\title{
A 3-3-1 model with low scale seesaw mechanisms
}

\author{
A. E. Cárcamo Hernández ${ }^{\mathrm{a}}$, Yocelyne Hidalgo Velásquez ${ }^{\mathrm{b}}$, Nicolás A. Pérez-Julve ${ }^{\mathrm{c}}$ \\ Universidad Técnica Federico Santa María and Centro Científico-Tecnológico de Valparaíso, Casilla 110-V, Valparaíso, Chile
}

Received: 2 July 2019 / Accepted: 19 September 2019 / Published online: 8 October 2019

(C) The Author(s) 2019

\begin{abstract}
We construct a viable 3-3-1 model with two $S U(3)_{L}$ scalar triplets, extended fermion and scalar spectrum, based on the $T^{\prime}$ family symmetry and other auxiliary cyclic symmetries, whose spontaneous breaking yields the observed pattern of SM fermion mass spectrum and fermionic mixing parameters. In our model the SM quarks lighter than the top quark, get their masses from a low scale Universal seesaw mechanism, the SM charged lepton masses are produced by a Froggatt-Nielsen mechanism and the small light active neutrino masses are generated from an inverse seesaw mechanism. The model is consistent with the low energy SM fermion flavor data and successfully accommodates the current Higgs diphoton decay rate and predicts charged lepton flavor violating decays within the reach of the forthcoming experiments.
\end{abstract}

\section{Introduction}

The existence of three fermion families and the observed pattern of Standard Model (SM) fermion masses and mixing angles are not explained within the context of the SM. Whereas in the quark sector, the mixing angles are small, in the lepton sector two of the mixing angles are large and one is small, of the order of the Cabbibo angle. The pattern of SM fermion masses is extended over a range of 5 orders of magnitude in the quark sector and a dramatically broader range of about 13 orders of magnitude, when the light active neutrino sector is included. That flavour puzzle of the SM motivates the study of theories with an extended particle spectrum and enlarged symmetries, whose spontaneous breaking produces the observed SM fermion mass and mixing hierarchy.

In addition, the SM predicts very tiny branching ratios for the charged lepton flavor violating processes (cLFV)

\footnotetext{
a e-mail: antonio.carcamo@usm.cl

b e-mail: yocehidalgov@gmail.com

c e-mail: nicolasperezjulve@gmail.com
}

$\mu \rightarrow e \gamma, \tau \rightarrow \mu \gamma$ and $\tau \rightarrow e \gamma$, several orders of magnitude below their corresponding projective experimental sensitivity. On the other low scale seesaw models [1-6] predict branching ratios for the cLFV processes within the reach of the projective experimental sensitivity. Thus, a future observation of charged lepton flavor violating processes will provide an undubitable evidence of Physics Beyond the Standard Model and will shed light on the dynamics that produces tiny light active neutrino masses and the measured leptonic mixing angles.

Furthermore, the origin of the family structure of fermions, which is not addressed by the SM, can be explained in theories having an extended $S U(3)_{C} \times S U(3)_{L} \times U(1)_{X}$ gauge symmetry, called 3-3-1 models [7-53]. In these models, the cancellation of chiral anomalies takes place when the number of $S U(3)_{L}$ fermionic triplets is equal to the number of $S U(3)_{L}$ fermionic antitriplets, which happens when the number of fermion generations is a multiple of three. Furthermore, when combined with the QCD asymptotic freedom, the 3-3-1 models predict that the number of fermion generations is exactly three. In addition, the nonuniversal $U(1)_{X}$ charge assignments for the left handed quarks fields in the 3-3-1 models, are crucial for explaining the large mass splitting between the heaviest quark and the two lighter ones. Other phenomenological advantages of the 3-3-1 models are: (1) they address the electric charge quantization [54,55], (2) they contain several sources of CP violation $[56,57]$, (3) they have a natural Peccei-Quinn symmetry, thus allowing to address the strong-CP problem [58-61], (4) the 33-1 models with heavy sterile neutrinos in the fermionic spectrum have cold dark matter candidates as weakly interacting massive particles (WIMPs) [62-65], (5) they predict the bound $\sin \theta_{W}^{2}<\frac{1}{4}$, for the weak mixing parameter, (6) the 3-3-1 models with three right handed Majorana neutrinos and non SM fermions without non SM electric charges, allow the implementation of a low scale seesaw mechanism, which could be inverse or linear, thus allowing to explain the smallness of the light active neutrinos masses and to predict 
charged lepton flavor violating process within the reach of the forthcoming experiments.

In this work, motivated by the aforementioned considerations, we propose a 3-3-1 model with two $S U(3)_{L}$ scalar triplets, extended fermion and scalar spectrum, consistent with SM fermion masses and mixings. Our model incorporates a Universal low scale seesaw mechanism to generate the masses for the SM quarks lighter than the top quark, a Froggatt-Nielsen mechanism that produces the SM charged lepton masses and an inverse seesaw mechanism that gives rise to small light active neutrino masses. In our model we use the $T^{\prime}$ symmetry, which in combination with other auxiliary symmetries, allows a viable description of the current SM fermion mass spectrum and mixing parameters. We use the double tetrahedral group $T^{\prime}$ since it is the smallest discrete subgroup of $S U(2)$ as well as the smallest group of any kind with 1-, 2- and 3-dimensional representations and the multiplication rule $\mathbf{2} \otimes \mathbf{2}=\mathbf{3} \oplus \mathbf{1}$, thus allowing to reproduce the successful $U(2)$ textures [66]. Note that the discrete group $T^{\prime}$ [49,66-85], together with the groups $A_{4}$ [37,45,86-126], $S_{4}$ $[16,92,125,127-145,145-148]$ and $\Delta(27)[48,149-174]$, is the smallest group containing an irreducible triplet representation that can accommodate the three fermion families of the Standard model (SM). These groups have attracted a lot of attention of the model building community since they successfully describe the observed SM fermion mass spectrum and mixing parameters.

The content of this paper goes as follows. In Sect. 2.1 we outline the proposed model, describing its fermionic and scalar spectrum as well as their assignments under the different continuous and discrete groups. The gauge sector of the model is discussed in Sect. 2.2, whereas the scalar potential for two $S U(3)_{L}$ triplets is discussed in Sect. 2.3. The implications of our model in SM quark masses and mixings are discussed in Sect. 3. In Sect. 4, we present our results in terms of lepton masses and mixing, which is followed by a numerical analysis. The implications of our model in the Higgs diphoton decay rate are discussed in Sect. 5. In Sect. 6, lepton flavor violating decays of the charged leptons are discussed, where sterile neutral lepton masses are constrained. Conclusions are given in Sect. 7. Some technical details are shown in the appendices: "Appendix A" provides a description of the $T^{\prime}$ discrete group. "Appendix B" includes a discussion of the scalar potential for a $T^{\prime}$ scalar triplet and its minimization condition.

\section{The model}

\subsection{Particle content}

We consider a model based on the extended gauge symmetry $S U(3)_{C} \times S U(3)_{L} \times U(1)_{X}(3-3-1$ model $)$ which is sup- plemented by the $U(1)_{L_{g}}$ global lepton number symmetry and the $T^{\prime} \times Z_{6} \times Z_{8} \times Z_{12}$ discrete group. Our model is an extension of the 3-3-1 model with two $S U(3)_{L}$ scalar triplets, where the scalar sector is augmented by the inclusion of several gauge singlet scalars and the fermion spectrum is enlarged by adding several vector like fermions and right handed Majorana neutrinos. The $S U(3)_{L}$ singlet vector like fermions are introduced in our model in order to implement a Universal Seesaw mechanism [175-177] for the generation of the masses of SM quarks lighter than the top quark. We additionally introduce three gauge singlet right handed Majorana neutrinos which are crucial to incorporate the inverse seesaw mechanism in our model. In our model the non SM fermions do not have non SM electric charges, thus implying that the third component of the $S U(3)_{L}$ leptonic triplet is electrically neutral, thus allowing the implementation of an inverse seesaw mechanism [4,178-183] to generate the small light active neutrino masses. The SM charged lepton masses are produced from a Froggatt-Nielsen mechanism [184], which is triggered by non renormalizable Yukawa interactions involving the $S U(3)_{L}$ scalar triplets $\eta$ and $\chi$ as well as several gauge singlet scalars charged under the different discrete group factors of the model. In our model the hierarchy of SM charged fermion masses and fermionic mixing parameters is produced by the spontaneous breaking of the $T^{\prime} \times Z_{6} \times Z_{8} \times Z_{12}$ discrete group. The $S U(3)_{C} \times S U(3)_{L} \times U(1)_{X} \times T^{\prime} \times Z_{6} \times Z_{8} \times Z_{12}$ assignments of the scalar and fermionic fields of our model are shown in Tables 1 and 2, respectively. Notice that in these tables the dimensions of the $S U(3)_{C}, S U(3)_{L}$ and $T^{\prime}$ representations are specified by the numbers in boldface and the different $Z_{N}$ charges are written in additive notation. Let us note that a field $\psi$ transforms under the $Z_{N}$ symmetry with a corresponding $q_{n}$ charge as: $\psi \rightarrow e^{\frac{2 \pi i q_{n}}{N}} \psi, n=0,1,2,3 \cdots N-1$. An explanation of the role of the different discrete group factors of the model is provided in the following. The double tetrahedral group $T^{\prime}$ selects the allowed entries of the mass matrices for SM charged fermions and neutrinos, thus allowing a reduction of the model parameters. In addition, as it will be shown below in Sects. 3 and 4, the spontaneous breaking of the $T^{\prime}$ discrete group will be crucial to generate the observed $\mathrm{CP}$ violation in both quark and lepton sectors, without the need of invoking complex Yukawa couplings. Let us note that $T^{\prime}$ is the smallest discrete subgroup of $S U(2)$ as well as the smallest group of any kind with 1-, 2- and 3-dimensional representations and the multiplication rule $\mathbf{2} \otimes \mathbf{2}=\mathbf{3} \oplus \mathbf{1}$, thus allowing to reproduce the successful $U(2)$ textures as pointed out in Ref. [66]. The $Z_{6}$ discrete group separates the $T^{\prime}$ scalar triplets $(\rho, \phi$ and $\zeta)$ participating in the charged lepton Yukawa interactions from the one $(\xi)$ appearing in the neutrino Yukawa terms, thus allowing to treat the charged lepton and neutrino sectors independently. The $Z_{8}$ discrete 
group contributes in generating small lepton number violating Majorana mass terms that yields a small $\mu$ parameter of the inverse seesaw mechanism that produces the tiny light active neutrino masses. Furthermore, $Z_{8}$ discrete group helps in shaping the texture for the SM charged leptons, that allows a reduction of the model parameters. The $Z_{12}$ discrete group is crucial for: (1) explaining the SM charged lepton mass hierarchy, (2) shaping the hierarchical structure of the quark mass matrices necessary to get a realistic pattern of quark masses and mixing and (3) generating small lepton number violating Majorana mass terms thus allowing to provide a natural explanation for the tiny values of the light active neutrino masses.

The full symmetry $\mathcal{G}$ of our model features the following two-step spontaneous breaking:

$$
\begin{aligned}
\mathcal{G}= & S U(3)_{C} \times S U(3)_{L} \times U(1)_{X} \times U(1)_{L_{g}} \\
& \times T^{\prime} \times Z_{6} \times Z_{8} \times Z_{12} \\
& \stackrel{\Lambda_{\text {int }}}{\longrightarrow} S U(3)_{C} \times S U(2)_{L} \times U(1)_{Y} \times Z_{2}^{\left(L_{g}\right)} \\
& \stackrel{v_{\eta}}{\longrightarrow} S U(3)_{C} \times U(1)_{Q} \times Z_{2}^{\left(L_{g}\right)}
\end{aligned}
$$

where the symmetry breaking scales fulfill the hierarchy $\Lambda_{\text {int }} \sim v_{\chi} \gg v_{\eta}$. It is worth mentioning that the first step of symmetry breaking in Eq. (2.1) is triggered by the $S U(3)_{L}$ scalar triplet $\chi$, whose third component acquires a 10 $\mathrm{TeV}$ scale vacuum expectation value (VEV) that breaks the $S U(3)_{L} \times U(1)_{X}$ gauge symmetry as well as by the $S U(3)_{L}$ scalar singlets whose VEVs break the $T^{\prime} \times Z_{6} \times Z_{8} \times Z_{12}$ discrete group. The non SM particles get masses at the $v_{\chi}$ scale after the spontaneous breaking of the $S U(3)_{L} \times U(1)_{X}$ gauge symmetry. We consider $v_{\chi} \sim \mathcal{O}(10) \mathrm{TeV}$, because the experimental data on $K, D$ and $B$ meson mixings set a lower bound of about $4 \mathrm{TeV}$ [185] for the $Z^{\prime}$ gauge boson mass in 3-3-1 models, which translates in a lower limit of about $10 \mathrm{TeV}$ for the $S U(3)_{L} \times U(1)_{X}$ gauge symmetry breaking scale $v_{\chi}$. In addition, $v_{\chi} \sim \mathcal{O}(10) \mathrm{TeV}$ is also consistent with the collider constraints as well as with the constraints that the decays $B_{s, d} \rightarrow \mu^{+} \mu^{-}$and $B_{d} \rightarrow K^{*}(K) \mu^{+} \mu^{-}$ impose on the $Z^{\prime}$ masses. It is worth mentioning that the LHC searches constrain the $Z^{\prime}$ gauge boson mass in 3-3-1 models to be larger than about $2.5 \mathrm{TeV}$ [186], which corresponds to a lower limit of $6.3 \mathrm{TeV}$ for the $S U(3)_{C} \times S U(3)_{L} \times U(1)_{X}$ symmetry breaking scale $v_{\chi}$. On the other hand, the decays $B_{s, d} \rightarrow \mu^{+} \mu^{-}$and $B_{d} \rightarrow K^{*}(K) \mu^{+} \mu^{-}$set lower limits on the $Z^{\prime}$ gauge boson mass ranging from $1 \mathrm{TeV}$ up to $3 \mathrm{TeV}$ $[15,187-190]$. Consequently, the scale $v_{\chi} \sim \mathcal{O}(10) \mathrm{TeV}$ is consistent with the aforementioned constraints. Furthermore, we assume that the discrete symmetries of the model are broken at the same scale of breaking of the $S U(3)_{L} \times U(1)_{X}$ gauge symmetry. Moreover, let us note that the second step of symmetry breaking in Eq. (2.1) is triggered by the $S U(3)_{L}$ scalar triplet $\eta$, whose first component get a VEV that sat- isfies $v_{\eta}=v=246 \mathrm{GeV}$ and provides masses for the SM particles. Note that the $U(1)_{L_{g}}$ global lepton number symmetry is assumed to be spontaneously broken down to a residual discrete $Z_{2}^{\left(L_{g}\right)}$ by the vacuum expectation value (VEV) of the $U(1)_{L_{g}}$ charged gauge-singlet scalar $\varphi$, having a nontrivial $U(1)_{L_{g}}$ charge, as indicated by Table 1 . The residual discrete $Z_{2}^{\left(L_{g}\right)}$ lepton number symmetry, under which the leptons are charged and the other particles are neutral, forbids interactions involving an odd number of leptons, thus preventing proton decay. The corresponding massless Goldstone boson, namely, the Majoron, is phenomenologically harmless since it is a $S U(3)_{L}$ scalar singlet.

Given that we are considering a 3-3-1 model where the non SM fermions do not have exotic electric charges, the electric charge in our model is defined in terms of the $S U(3)$ generators and the identity as follows:

$Q=T_{3}+\beta T_{8}+X I=T_{3}-\frac{1}{\sqrt{3}} T_{8}+X I$,

with $I=\operatorname{diag}(1,1,1), T_{3}=\frac{1}{2} \operatorname{diag}(1,-1,0)$ and $T_{8}=$ $\left(\frac{1}{2 \sqrt{3}}\right) \operatorname{diag}(1,1,-2)$ for a $S U(3)_{L}$ triplet. Furthermore, the lepton number has a gauge component as well as a complementary global one, as indicated by the following relation:

$L=\frac{4}{\sqrt{3}} T_{8}+L_{g}$,

being $L_{g}$ a conserved charge associated with the $U(1)_{L_{g}}$ global lepton number symmetry.

The $S U(3)_{L}$ triplet scalar fields $\chi$ and $\eta$ can be expanded around the minimum as follows:

$$
\begin{aligned}
& \chi=\left(\begin{array}{c}
\chi_{1}^{0} \\
\chi_{2}^{-} \\
\frac{1}{\sqrt{2}}\left(v_{\chi}+\xi_{\chi} \pm i \zeta_{\chi}\right)
\end{array}\right), \\
& \eta=\left(\begin{array}{c}
\frac{1}{\sqrt{2}}\left(v_{\eta}+\xi_{\eta} \pm i \zeta_{\eta}\right) \\
\eta_{2}^{-} \\
\eta_{3}^{0}
\end{array}\right) .
\end{aligned}
$$

The $S U(3)_{L}$ fermionic triplets and antitriplets can be represented as:

$$
\begin{aligned}
Q_{n L} & =\left(\begin{array}{c}
D_{n} \\
-U_{n} \\
J_{n}
\end{array}\right)_{L}, \quad Q_{3 L}=\left(\begin{array}{c}
U_{3} \\
D_{3} \\
T
\end{array}\right)_{L}, \\
L_{i L} & =\left(\begin{array}{c}
v_{i} \\
e_{i} \\
v_{i}^{c}
\end{array}\right)_{L}, \quad n=1,2, \quad i=1,2,3 .
\end{aligned}
$$

With the particle content shown in Tables 1 and 2, the following relevant Yukawa terms for the quark and lepton sector invariant under the group $\mathcal{G}$ arise:

$$
-\mathcal{L}_{Y}^{(q)}=y^{(T)} \bar{Q}_{3 L} \chi T_{R}+y^{(J)}\left(\bar{Q}_{L} \chi^{*} J_{R}\right)_{1}
$$


Table 1 Scalar assignments under $S U(3)_{C} \times S U(3)_{L} \times$ $U(1)_{X} \times T^{\prime} \times Z_{6} \times Z_{8} \times Z_{12}$

\begin{tabular}{llllllllllllllll}
\hline & $\chi$ & $\eta$ & $\varphi$ & $\sigma$ & $\xi$ & $\rho$ & $\phi$ & $\zeta$ & $S_{1}$ & $S_{2}$ & $S_{3}$ & $S_{4}$ & $S_{5}$ & $S_{6}$ \\
\hline$S U(3)_{C}$ & $\mathbf{1}$ & $\mathbf{1}$ & $\mathbf{1}$ & $\mathbf{1}$ & $\mathbf{1}$ & $\mathbf{1}$ & $\mathbf{1}$ & $\mathbf{1}$ & $\mathbf{1}$ & $\mathbf{1}$ & $\mathbf{1}$ & $\mathbf{1}$ & $\mathbf{1}$ & $\mathbf{1}$ \\
$S U(3)_{L}$ & $\mathbf{3}$ & $\mathbf{3}$ & $\mathbf{1}$ & $\mathbf{1}$ & $\mathbf{1}$ & $\mathbf{1}$ & $\mathbf{1}$ & $\mathbf{1}$ & $\mathbf{1}$ & $\mathbf{1}$ & $\mathbf{1}$ & $\mathbf{1}$ & $\mathbf{1}$ & $\mathbf{1}$ \\
$U(1)_{X}$ & $-\frac{1}{3}$ & $-\frac{1}{3}$ & 0 & 0 & 0 & 0 & 0 & 0 & 0 & 0 & 0 & 0 & 0 & 0 \\
$U(1)_{L_{g}}$ & $\frac{4}{3}$ & $-\frac{2}{3}$ & 2 & 0 & 0 & 0 & 0 & 0 & 0 & 0 & 0 & 0 & 0 & 0 \\
$T^{\prime}$ & $\mathbf{1}$ & $\mathbf{1}$ & $\mathbf{1}^{\prime}$ & $\mathbf{1}$ & $\mathbf{3}$ & $\mathbf{3}$ & $\mathbf{3}$ & $\mathbf{3}$ & $\mathbf{2}$ & $\mathbf{2}^{\prime}$ & $\mathbf{2}^{\prime}$ & $\mathbf{1}$ & $\mathbf{1}^{\prime}$ & $\mathbf{1}^{\prime \prime}$ \\
$Z_{6}$ & 0 & 0 & 0 & 0 & 0 & 2 & 2 & 2 & 0 & 0 & 0 & 3 & 0 & 0 \\
$Z_{8}$ & 0 & 0 & -4 & 0 & 0 & -1 & -2 & 0 & 0 & 0 & 0 & 0 & 1 & 1 \\
$Z_{12}$ & 0 & 0 & -4 & -1 & 0 & 0 & 0 & 0 & 0 & 0 & 1 & 0 & -1 & 0 \\
\hline
\end{tabular}

$+y_{3}^{(U)} \bar{Q}_{3 L} \eta U_{3 R}+y_{\widetilde{T}}\left(\widetilde{T}_{L} S_{4} \widetilde{T}_{R}\right)_{1}$

$+\sum_{j=1}^{3}\left(m_{B}\right)_{j} \bar{B}_{j L} B_{j R}$

$+x_{11}^{(U)} \widetilde{\widetilde{T}}_{L} S_{1} U_{1 R} \frac{\sigma^{5}}{\Lambda^{5}}+x_{22}^{(U)} \widetilde{\widetilde{T}}_{L} S_{2} U_{2 R} \frac{S_{5}}{\Lambda}$

$+x_{12}^{(U)} \widetilde{T}_{L} S_{1} U_{2 R} \frac{S_{6} \sigma}{\Lambda^{2}}+y^{(U)} \varepsilon_{a b c}\left(\bar{Q}_{L}^{a} \eta^{b} \chi^{c} \widetilde{T}_{R}\right)_{1} \frac{1}{\Lambda}$

$+x_{j}^{(D)} \bar{B}_{j L} S_{4} D_{j R} \frac{\sigma^{2}}{\Lambda^{2}}+y_{11}^{(D)} \bar{Q}_{L} \eta^{*} B_{1 R} \frac{S_{1} \sigma^{2}}{\Lambda^{3}}$

$+y_{22}^{(D)} \bar{Q}_{L} \eta^{*} B_{2 R} \frac{S_{2}^{*}}{\Lambda}+y_{23}^{(D)} \bar{Q}_{L} \eta^{*} B_{3 R} \frac{S_{2}}{\Lambda}$

$+y_{13}^{(D)} \bar{Q}_{L} \eta^{*} B_{3 R} \frac{S_{3} \sigma}{\Lambda^{2}}$

$+y_{33}^{(D)} \varepsilon_{a b c} \bar{Q}_{3 L}^{a}\left(\eta^{*}\right)^{b}\left(\chi^{*}\right)^{c} B_{3 R} \frac{1}{\Lambda}+H . c$,

$-\mathcal{L}_{Y}^{(l)}=y_{1}^{(L)} \varepsilon_{a b c}\left(\bar{L}_{L}^{a}\left(\eta^{*}\right)^{b}\left(\chi^{*}\right)^{c} \rho\right)_{1} e_{1 R} \frac{\sigma^{6} S_{6}}{\Lambda^{9}}$

$+y_{2}^{(L)} \varepsilon_{a b c}\left(\bar{L}_{L}^{a}\left(\eta^{*}\right)^{b}\left(\chi^{*}\right)^{c} \phi\right)_{1^{\prime \prime}} e_{2 R} \frac{\sigma^{3}}{\Lambda^{5}}$

$+\frac{y_{3}^{(L)}}{\Lambda^{2}} \varepsilon_{a b c}\left(\bar{L}_{L}^{a}\left(\eta^{*}\right)^{b}\left(\chi^{*}\right)^{c} \zeta\right)_{1^{\prime}} e_{3 R} \frac{S_{6}}{\Lambda}$

$+y_{\rho} \varepsilon_{a b c} \varepsilon_{d e c}\left(\bar{L}_{L}^{a}\left(L_{L}^{C}\right)^{b}\right)_{\mathbf{3}_{2}} \eta^{d} \chi^{e} \frac{\xi \sigma^{2}}{\Lambda^{4}}$

$+y_{\chi}^{(L)}\left(\bar{L}_{L} \chi N_{R}\right)_{1}$

$+h_{1 N}\left(N_{R} \overline{N_{R}^{C}}\right)_{1} \varphi \frac{\left(\sigma^{*}\right)^{6} S_{6}^{4}}{\Lambda^{10}}$

$+h_{2 N}\left(N_{R} \overline{N_{R}^{C}}\right)_{\mathbf{3}_{1}} \varphi \frac{\xi\left(\sigma^{*}\right)^{6} S_{6}^{4}}{\Lambda^{11}}+$ H.c.,

where the dimensionless couplings in Eqs. (2.6) and (2.7) are $\mathcal{O}(1)$ parameters.

As shown in detail in the "Appendix B", the following VEV patterns for the $T^{\prime}$ scalar triplets are consistent with the scalar potential minimization equations for a large region of parameter space:

$$
\begin{aligned}
\langle\rho\rangle= & v_{\rho}\left(e^{-i \alpha}\left(\cos \gamma-e^{i\left(2 \phi_{1}+\phi_{2}\right)} \sin \gamma\right),\right. \\
& \left.\times 1, e^{i \alpha}\left(\cos \gamma-e^{-i\left(2 \phi_{1}+\phi_{2}\right)} \sin \gamma\right)\right),
\end{aligned}
$$

$$
\begin{aligned}
\langle\phi\rangle= & v_{\phi}\left(1, e^{i \alpha}\left(\cos \gamma+e^{i\left(\phi_{2}-\phi_{1}\right)} \sin \gamma\right)\right. \\
& \left.\times e^{-i \alpha}\left(\cos \gamma+e^{-i\left(\phi_{2}-\phi_{1}\right)} \sin \gamma\right)\right) \\
\langle\zeta\rangle= & v_{\zeta}\left(e^{i \alpha}(\cos \gamma+\sin \gamma), e^{-i \alpha}(\cos \gamma+\sin \gamma), 1\right) \\
& \times\langle\xi\rangle=\frac{v_{\xi}}{\sqrt{3}}(1,1,1) .
\end{aligned}
$$

In what regards the $T^{\prime}$ scalar doublets, we consider the following VEV configurations, which are natural solutions of the scalar potential minimization conditions:

$$
\left\langle S_{1}\right\rangle=v_{S_{1}}(0,1), \quad\left\langle S_{2}\right\rangle=v_{S_{2}}(-1,0), \quad\left\langle S_{3}\right\rangle=v_{S_{3}}(0,1) .
$$

Furthermore, since the observed pattern of the SM charged fermion masses and quark mixing angles is produced by the spontaneous breaking of the $T^{\prime} \times Z_{6} \times Z_{8} \times Z_{12}$ discrete group, we set the VEVs of the $S U(3)_{L}$ singlet scalar fields with respect to the Wolfenstein parameter $\lambda=0.225$ and the model cutoff $\Lambda$, as follows:

$$
\begin{aligned}
v_{S_{k}} \sim v_{\eta} \sim \lambda^{3} \Lambda \ll & v_{\chi} \sim v_{\zeta} \sim v_{\rho} \sim v_{\varphi} \sim v_{\sigma} \\
& \sim v_{\xi} \sim v_{\phi} \sim v_{S_{l}} \\
& \sim \Lambda_{\text {int }}=\lambda \Lambda, \quad k=1,2,3, \quad l=4,5,6 .
\end{aligned}
$$

Considering $\Lambda_{\text {int }} \sim \mathcal{O}(10) \mathrm{TeV}$, from Eq. (2.10) we find for the model cutoff the estimate $\Lambda \sim \mathcal{O}(40) \mathrm{TeV}$.

\subsection{The gauge sector}

The gauge bosons associated with the group $S U(3)_{L}$ for the case $\beta=-1 / \sqrt{3}$ are written as follows:

$\mathbf{W}_{\mu}=W_{\mu}^{\alpha} G_{\alpha}$

$$
=\frac{1}{2}\left(\begin{array}{ccc}
W_{\mu}^{3}+\frac{1}{\sqrt{3}} W_{\mu}^{8} & \sqrt{2} W_{\mu}^{+} & \sqrt{2} K_{\mu}^{0} \\
\sqrt{2} W_{\mu}^{-} & -W_{\mu}^{3}+W_{\mu}^{8} & \sqrt{2} K_{\mu}^{-} \\
\sqrt{2} K_{\mu}^{0} & \sqrt{2} K_{\mu}^{+} & -\frac{2}{\sqrt{3}} W_{\mu}^{8}
\end{array}\right),
$$




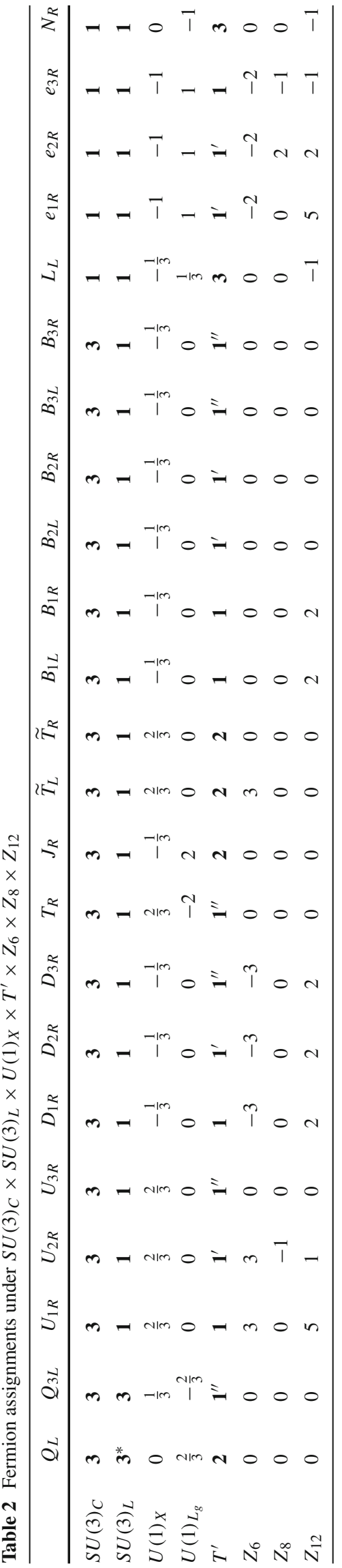

where the electric charges of each gauge field correspond to the entries of the matrix:

$Q_{W}=\left(\begin{array}{ccc}0 & 1 & 0 \\ -1 & 0 & -1 \\ 0 & 1 & 0\end{array}\right)$

The gauge field associated with the $U(1)_{X}$ symmetry is electrically neutral, i.e., it has $Q_{B}=0$ and is represented as follows:

$\mathbf{B}_{\mu}=\mathbf{I}_{3 \times 3} B_{\mu}$.

The gauge sector associated with the $S U(3)_{L} \times U(1)_{X}$ group of the 3-3-1 models, is composed of five electrically neutral and four electrically charged gauge bosons. In the gauge boson spectrum there is one massless electrically neutral gauge boson which corresponds to the photon and eight massive gauge boson fields, namely, $Z, W^{ \pm}, Z^{\prime}$, $W^{\prime \pm}, \bar{K}^{0}, K^{0}$. Five of the massive gauge bosons, $Z^{\prime}, W^{\prime \pm}$, $\bar{K}^{0}, K^{0}$ acquire their masses after the spontaneous breaking of the $S U(3)_{L} \times U(1)_{X}$ gauge symmetry down to the $S U(2)_{L} \times U(1)_{Y}$, whereas the $Z$ and $W^{ \pm}$gauge bosons become massive after electroweak symmetry breaking.

The gauge boson mass terms as well as interactions between the scalar and gauge bosons arise from the following kinetic term:

$$
\begin{aligned}
\mathcal{L}_{K}= & \sum_{\Phi=\eta, \chi}\left(D^{\mu} \Phi\right)^{\dagger}\left(D_{\mu} \Phi\right) \\
= & \sum_{\Phi=\eta, \chi}[\overbrace{\left(\partial^{\mu} \Phi\right)^{\dagger}\left(D_{\mu} \Phi\right)+\left(D^{\mu} \Phi\right)^{\dagger}\left(\partial_{\mu} \Phi\right)}^{(1)}-\left(\partial^{\mu} \Phi\right)^{\dagger}\left(\partial_{\mu} \Phi\right) \\
& +\overbrace{\Phi^{\dagger}\left(g W^{\mu}+g^{\prime} X_{\Phi} B^{\mu}\right)^{\dagger}\left(g W^{\mu}+g^{\prime} X_{\Phi} B^{\mu}\right) \Phi}^{(2)}],
\end{aligned}
$$

where the covariant derivative in 3-3-1 models is defined as follows [191]:

$D_{\mu}=\partial_{\mu}+i g W_{\mu}^{\alpha} G_{\alpha}+i g^{\prime} X_{\Phi} B_{\mu}$.

Notice that the first two terms of Eq. (2.14), which are denoted as (1), include the couplings between the gauge bosons and the derivatives of the scalar fields, thus allowing to get information about each would-be Goldstone boson interacting with its corresponding massive gauge boson. In addition, the last term of Eq. (2.14), which is denoted as (2), contains information about the masses of the gauge bosons and its couplings with the physical scalar fields. 
Table 3 Physical gauge bosons mass spectrum

\begin{tabular}{ll}
\hline Gauge Boson & Squared mass \\
\hline$W^{ \pm}$ & $\frac{1}{4} g^{2} v_{\eta}^{2}$ \\
$W^{ \pm}$ & $\frac{1}{4} g^{2} v_{\chi}^{2}$ \\
$\gamma$ & 0 \\
$Z$ & $\frac{1}{9}\left(\Xi_{1}-\Xi_{2}\right)$ \\
$Z^{\prime}$ & $\frac{1}{9}\left(\Xi_{1}+\Xi_{2}\right)$ \\
$K^{0}, \bar{K}^{0}$ & $\frac{g^{2}}{8}\left(v_{\chi}^{2}+v_{\eta}^{2}\right)$ \\
\hline
\end{tabular}

The different entries of the gauge boson squared mass matrices are obtained from the following relation:

$M_{V_{i} V_{j}}^{2}=\frac{\partial^{2} \mathcal{L}_{K}}{\partial V_{i} \partial V_{j}}$

where for the charged gauge bosons $V_{i}=W^{ \pm}, W^{\prime \pm}$, whereas for the neutral ones $V_{i}=W^{3}, W^{8}, B, \bar{K}^{0}, K^{0}$. Then, the squared mass matrices for the charged and neutral gauge bosons are respectively given by:

$M_{\text {charged }}^{2}=\left(\begin{array}{cc}\frac{1}{4} g^{2} v_{\eta}^{2} & 0 \\ 0 & \frac{1}{4} g^{2} v_{\chi}^{2}\end{array}\right)$,

$M_{\text {neutral }}^{2}=\left(\begin{array}{cccc}\frac{1}{4} g^{2} v_{\eta}^{2} & \frac{g^{2} v_{\eta}^{2}}{4 \sqrt{3}} & -\frac{1}{6} g v_{\eta}^{2} g^{\prime} & 0 \\ \frac{g^{2} v_{\eta}^{2}}{4 \sqrt{3}} & \frac{1}{12} g^{2} v_{\eta}^{2}+\frac{1}{3} g^{2} v_{\chi}^{2} & \frac{g v_{\chi}^{2} g^{\prime}}{3 \sqrt{3}}-\frac{g v_{\eta}^{2} g^{\prime}}{6 \sqrt{3}} & 0 \\ -\frac{1}{6} g v_{\eta}^{2} g^{\prime} & \frac{g v_{\chi}^{2} g^{\prime}}{3 \sqrt{3}}-\frac{g v_{\eta}^{2} g^{\prime}}{6 \sqrt{3}} & \frac{1}{9} v_{\eta}^{2}\left(g^{\prime}\right)^{2}+\frac{1}{9} v_{\chi}^{2}\left(g^{\prime}\right)^{2} & 0 \\ 0 & 0 & 0 & \frac{1}{8} g^{2} v_{\eta}^{2}+\frac{1}{8} g^{2} v_{\chi}^{2}\end{array}\right)$.

The gauge bosons mass spectrum of the model is summarized in Table 3

$$
\begin{aligned}
& \Xi_{1}=3 g^{2}\left(v_{\eta}^{2}+v_{\chi}^{2}\right)+\left(g^{\prime}\right)^{2}\left(v_{\eta}^{2}+v_{\chi}^{2}\right), \\
& \Xi_{2}=\sqrt{\left(3 g^{2}+\left(g^{\prime}\right)^{2}\right)^{2}\left(v_{\eta}^{2}+v_{\chi}^{2}\right)^{2}-9 g^{2}\left(3 g^{2}+4\left(g^{\prime}\right)^{2}\right) v_{\eta}^{2} v_{\chi}^{2}} .
\end{aligned}
$$

The squared masses for the $Z$ and $Z^{\prime}$ gauge bosons can be approximatelly written as [13]:

$$
\begin{aligned}
M_{Z}^{2} & =\frac{g^{2}}{4 c_{W}} v_{\eta}^{2}, \\
M_{Z^{\prime}}^{2} & =\frac{g^{2}}{3-4 s_{W}^{2}} v_{\chi}^{2},
\end{aligned}
$$

where $c_{W}=\cos \theta_{W}, s_{W}=\sin \theta_{W}$ and $v_{\eta}=246 \mathrm{GeV}$. Consequently, for $v_{\chi} \approx 10 \mathrm{TeV}$, we find that the heavy gauge bosons have the masses $M_{W^{\prime}} \approx 3.3 \mathrm{TeV}$ and $M_{Z^{\prime}} \approx 4.5 \mathrm{TeV}$.

\subsection{Scalar potential for two $S U(3)_{L}$ scalar triplets}

For the sake of simplicity we neglect the mixing terms between the $S U(3)_{L}$ scalar triplets and the gauge singlet scalars. Then, the scalar potential for two $S U(3)_{L}$ scalar triplets takes the form:

$$
\begin{aligned}
V= & -\mu_{\chi}^{2}\left(\chi^{\dagger} \chi\right)-\mu_{\eta}^{2}\left(\eta^{\dagger} \eta\right)+\lambda_{1}\left(\chi^{\dagger} \chi\right)\left(\chi^{\dagger} \chi\right) \\
& +\lambda_{2}\left(\eta^{\dagger} \eta\right)\left(\eta^{\dagger} \eta\right)+\lambda_{3}\left(\chi^{\dagger} \chi\right)\left(\eta^{\dagger} \eta\right) \\
& +\lambda_{4}\left(\chi^{\dagger} \eta\right)\left(\eta^{\dagger} \chi\right),
\end{aligned}
$$

where $\chi$ and $\eta$ are the $S U(3)_{L}$ scalar triplets acquiring vacuum expectation values (VEVs) in their third and first components, respectively. The minimization conditions of the aforementioned scalar potential yields the following relations:

$$
\begin{gathered}
\frac{\partial V}{\partial v_{\chi}}=\frac{1}{2} \lambda_{3} v_{\eta}^{2} v_{\chi}+\lambda_{1} v_{\chi}^{3}-\mu_{1}^{2} v_{\chi}=0, \\
\frac{\partial V}{\partial v_{\eta}}=\frac{1}{2} \lambda_{3} v_{\eta} v_{\chi}^{2}+\lambda_{2} v_{\eta}^{3}-\mu_{2}^{2} v_{\eta}=0 .
\end{gathered}
$$

Thus, the VEV patterns for the $S U(3)_{L}$ scalar triplets $\chi$ and $\eta$ are compatible with a global minimum of the scalar potential

$$
\left.\frac{1}{8} g^{2} v_{\eta}^{2}+\frac{1}{8} g^{2} v_{\chi}^{2}\right)
$$

of (2.21). Solving these equations, the mass parameters can be obtained:

$\begin{aligned} \mu_{\chi}^{2} & =\frac{1}{2} \lambda_{3} v_{\eta}^{2}+\lambda_{1} v_{\chi}^{2}, \\ \mu_{\eta}^{2} & =\frac{1}{2} \lambda_{3} v_{\chi}^{2}+\lambda_{2} v_{\eta}^{2},\end{aligned}$

replacing these mass parameters in the Higgs potential, the neutral and charged scalar mass spectrum resulting from the two $S U(3)_{L}$ scalar triplets can be obtained from the following relations:

$M_{\Phi_{i} \Phi_{j}}^{2}=\left.\frac{\partial^{2} V}{\partial \Phi_{i} \Phi_{j}}\right|_{\Phi_{i}=0}, \quad M_{\Phi_{i}^{*} \Phi_{j}}^{2}=\left.\frac{\partial^{2} V}{\partial \Phi_{i} \Phi_{j}}\right|_{\Phi_{i}=0}$,

for the neutral scalar masses $\Phi_{i}=\xi_{\chi}, \xi_{\eta}, \zeta_{\chi}, \zeta_{\eta}, \chi^{0}, \eta^{0}$ and charged scalar masses $\Phi_{i}=\chi^{ \pm}, \eta^{ \pm}$respectively. The scalar 
Table 4 Physical scalar mass spectrum

\begin{tabular}{ll}
\hline Scalars & Masses \\
\hline$G_{1}^{0}=\zeta_{\chi}$ & $M_{G_{1}^{0}}^{2}=0$ \\
$G_{2}^{0}=\zeta_{\eta}$ & $M_{G_{2}^{0}}^{2}=0$ \\
$h_{1}^{0}=C_{\alpha} \xi_{\chi}-S_{\alpha} \xi_{\eta}$ & $M_{h_{1}^{0}}^{2}=\Delta_{1}-\Delta_{2}$ \\
$H_{1}^{0}=S_{\alpha} \xi_{\chi}+C_{\alpha} \xi_{\eta}$ & $M_{H_{1}^{0}}^{2}=\Delta_{1}+\Delta_{2}$ \\
$G_{3}^{0}=-C_{\beta} \chi^{0}+S_{\beta} \eta^{0}$ & $M_{G_{3}^{0}}^{2}=0$ \\
$H_{2}^{0}=S_{\beta} \chi^{0}+C_{\beta} \eta^{0}$ & $M_{H_{2}^{0}}^{2}=\lambda_{4}\left(v_{\eta}^{2}+v_{\chi}^{2}\right)$ \\
$G_{1}^{ \pm}=\chi^{ \pm}$ & $M_{G_{1}^{ \pm}}^{2}=0$ \\
$G_{2}^{ \pm}=\eta^{ \pm}$ & $M_{G_{2}^{ \pm}}^{2}=0$
\end{tabular}

mass matrices are shown below:

$$
\begin{array}{rlrl}
M_{\zeta \zeta}^{2} & =0_{2 \times 2}, & & M_{\chi^{ \pm} \eta^{ \pm}}^{2}=0_{2 \times 2}, \\
M_{\chi^{0} \eta^{0}}^{2}=\left(\begin{array}{cc}
\lambda_{4} v_{\eta}^{2} & \lambda_{4} v_{\eta} v_{\chi} \\
\lambda_{4} v_{\eta} v_{\chi} & \lambda_{4} v_{\chi}^{2}
\end{array}\right), & M_{\xi \xi}^{2}=\left(\begin{array}{cc}
2 \lambda_{1} v_{\chi}^{2} & \lambda_{3} v_{\eta} v_{\chi} \\
\lambda_{3} v_{\eta} v_{\chi} & 2 \lambda_{2} v_{\eta}^{2}
\end{array}\right) .
\end{array}
$$

Finally, the physical scalar mass spectrum resulting from the $S U(3)_{L}$ scalar triplets $\eta$ and $\chi$ is summarized in Table 4.

$$
\begin{aligned}
\Delta_{1} & =\lambda_{2} v_{\eta}^{2}+\lambda_{1} v_{\chi}^{2}, \\
\Delta_{2} & =\sqrt{\lambda_{3}^{2} v_{\eta}^{2} v_{\chi}^{2}-2 \lambda_{1} \lambda_{2} v_{\eta}^{2} v_{\chi}^{2}+\lambda_{2}^{2} v_{\eta}^{4}+\lambda_{1}^{2} v_{\chi}^{4}}, \\
\tan (2 \alpha) & =\frac{\lambda_{3} v_{\eta} v_{\chi}}{\lambda_{1} v_{\chi}^{2}-\lambda_{2} v_{\eta}^{2}}, \\
\tan (\beta) & =\frac{v_{\chi}}{v_{\eta}}
\end{aligned}
$$

The physical scalar spectrum resulting from the two $S U(3)_{L}$ scalar triplets is composed of the following fields: $2 \mathrm{CP}$-even Higgs bosons $\left(h_{1}^{0}, H_{1}^{0}\right)$ and one neutral Higgs boson $\left(H_{2}^{0}\right)$. The scalar $h_{1}^{0}$ is identified with the SM-like $125 \mathrm{GeV}$ Higgs boson found at the LHC. It's noteworthy that the neutral Goldstone bosons $G_{1}^{0}, G_{2}^{0}, G_{3}^{0}$ and $\bar{G}_{3}^{0}$ are associated to the longitudinal components of the $Z, Z^{\prime}, K^{0}$ and $\bar{K}^{0}$ gauge bosons. Furthermore, the charged Goldstone bosons $G_{1}^{ \pm}$and $G_{2}^{ \pm}$are associated to the longitudinal components of the $W^{ \pm}$ and $W^{\prime \pm}$ gauge bosons respectively.

Finally to close this section, we briefly comment about the LHC signals of a $Z^{\prime}$ gauge boson. The heavy $Z^{\prime}$ gauge boson is mainly produced via Drell-Yan mechanism and its corresponding production cross section has been found to range from $85 \mathrm{fb}$ up to $10 \mathrm{fb}$ for $Z^{\prime}$ gauge boson masses between $4 \mathrm{TeV}$ and $5 \mathrm{TeV}$ and LHC center of mass energy $\sqrt{S}=13 \mathrm{TeV}$ [192]. Such $Z^{\prime}$ gauge boson after being produced will decay into pair of SM particles, with dominant decay mode into quark-antiquark pairs as shown in detail in Refs. [15,193]. The two body decays of the $Z^{\prime}$ gauge boson in 3-3-1 models have been studied in details in Refs. [193]. In particular, in Ref. [193] it has been shown the $Z^{\prime}$ decays into a lepton pair in 3-3-1 models have branching ratios of the order of $10^{-2}$, which implies that the total LHC cross section for the $p p \rightarrow Z^{\prime} \rightarrow l^{+} l^{-}$resonant production at $\sqrt{S}=13 \mathrm{TeV}$ will be of the order of $1 \mathrm{fb}$ for a $4 \mathrm{TeV} Z^{\prime}$ gauge boson, which is below its corresponding lower experimental limit arising from LHC searches [194]. A detailed study of the collider phenomenology of this model is beyond the scope of this paper and is left for future studies.

\section{Quark masses and mixings}

In this section, we show that our model is able to reproduce the observed pattern of SM quark masses and mixings. From the quark Yukawa terms, it follows that the up-type mass matrix in the basis $\left(\bar{u}_{1 L}, \bar{u}_{2 L}, \bar{u}_{3 L}, \bar{T}_{L}, \bar{T}_{1 L}, \bar{T}_{3 L}\right)$ versus $\left(u_{1 R}, u_{2 R}, u_{3 R}, T_{R}, \widetilde{T}_{1 R}, \widetilde{T}_{2 R}\right)$ takes the form:

$$
\begin{aligned}
& M_{U}=\left(\begin{array}{cccc}
0_{2 \times 2} & 0_{2 \times 1} & 0_{2 \times 1} & A_{U} \\
0_{1 \times 2} & m_{t} & 0 & 0_{1 \times 2} \\
0_{1 \times 2} & 0 & M_{T} & 0_{1 \times 2} \\
B_{U} & 0_{2 \times 1} & 0_{2 \times 1} & M_{\widetilde{T}}
\end{array}\right), \\
& A_{U}=y^{(U)} \frac{v v_{\chi}}{2 \Lambda}\left(\begin{array}{cc}
0 & 1 \\
-1 & 0
\end{array}\right) \text {, } \\
& B_{U}=\left(\begin{array}{cc}
x_{11}^{(U)}\left(\frac{v_{\sigma}}{\Lambda}\right)^{5} v_{S_{1}} & x_{12}^{(U)} \frac{v_{S_{6}} v_{\sigma}}{\Lambda^{2}} v_{S_{1}} \\
0 & x_{22}^{(U)} \frac{v_{S_{5}}}{\Lambda} v_{S_{2}}
\end{array}\right) \\
& =\left(\begin{array}{cc}
z_{11}^{(U)} \lambda^{4} & z_{12}^{(U)} \lambda \\
0 & z_{22}^{(U)}
\end{array}\right) \lambda v_{S_{1}}, \\
& M_{T}=m_{\widetilde{T}}\left(\begin{array}{cc}
0 & 1 \\
-1 & 0
\end{array}\right) \text {, } \\
& m_{t}=y_{3}^{(U)} \frac{v}{\sqrt{2}}, \quad m_{T}=y^{(T)} \frac{v_{\chi}}{\sqrt{2}},
\end{aligned}
$$

while the down type quark mass matrix written in the basis $\left(\bar{d}_{1 L}, \bar{d}_{2 L}, \bar{d}_{3 L}, \bar{J}_{1 L}, \bar{J}_{2 L}, \bar{B}_{1 L}, \bar{B}_{2 L}, \bar{B}_{3 L}\right)-\left(d_{1 R}, d_{2 R}, d_{3 R}\right.$, $\left.J_{1 R}, J_{2 R}, B_{1 R}, B_{2 R}, B_{3 R}\right)$ reads:

$$
\begin{aligned}
M_{D} & =\left(\begin{array}{ccc}
0_{3 \times 3} & 0_{3 \times 2} & A_{D} \\
0_{2 \times 3} & M_{J} & 0_{2 \times 3} \\
B_{D} & 0_{3 \times 2} & M_{B}
\end{array}\right), \\
A_{D} & =\left(\begin{array}{ccc}
y_{11}^{(D)} \frac{v_{S_{1}} v_{\sigma}^{2}}{\Lambda^{3}} & 0 & y_{13}^{(D)} \frac{v_{S_{3}} v_{\sigma}}{\Lambda} \\
0 & y_{22}^{(D)} \frac{v_{S_{2}}}{\Lambda} & y_{23}^{(D)} \frac{v_{S_{2}}}{\Lambda} \\
0 & 0 & y_{33}^{(D)} \frac{v_{X}}{\Lambda}
\end{array}\right) \\
\frac{v}{\sqrt{2}} & =\left(\begin{array}{ccc}
z_{11}^{(D)} \lambda^{5} & 0 & z_{13}^{(D)} \lambda^{4} \\
0 & z_{22}^{(D)} \lambda^{3} & z_{23}^{(D)} \lambda^{3} \\
0 & 0 & z_{3}^{(D)} \lambda
\end{array}\right) \frac{v}{\sqrt{2}},
\end{aligned}
$$




$$
\begin{aligned}
& B_{D}=\left(\frac{v_{\sigma}}{\Lambda}\right)^{2}\left(\begin{array}{ccc}
x_{1}^{(D)} & 0 & 0 \\
0 & x_{2}^{(D)} & 0 \\
0 & 0 & x_{3}^{(D)}
\end{array}\right) \\
& v_{S_{4}}=\left(\begin{array}{ccc}
z_{1}^{(D)} & 0 & 0 \\
0 & z_{2}^{(D)} & 0 \\
0 & 0 & z_{3}^{(D)}
\end{array}\right) \lambda^{2} v_{S_{4}}, \\
& M_{J}=y^{(J)} \frac{v_{\chi}}{\sqrt{2}}\left(\begin{array}{cc}
0 & 1 \\
-1 & 0
\end{array}\right), \quad M_{B}=\left(\begin{array}{ccc}
m_{B_{1}} & 0 & 0 \\
0 & m_{B_{2}} & 0 \\
0 & 0 & m_{B_{3}}
\end{array}\right) .
\end{aligned}
$$

Assuming that the exotic quarks have TeV scale masses, we find that the SM quarks (excepting the top quark) get their masses from a Universal seesaw mechanism mediated by the two exotic up-type and three exotic down-type quarks, $\widetilde{T}_{n}$ $(n=1,2)$ and $B_{i}(i=1,2,3)$, respectively. Due to the symmetries of the model, there are no mixing mass terms between the top quark and the remaining up-type quarks. Thus, the Universal Seesaw mechanism gives rise to the following SM quark mass matrices:

$$
\begin{aligned}
& \tilde{M}_{U}=\left(\begin{array}{cc}
A_{U} M_{\widetilde{T}}^{-1} B_{U} & 0_{2 \times 1} \\
0_{1 \times 2} & m_{t}
\end{array}\right) \\
& =\left(\begin{array}{ccc}
z_{11}^{(U)} y^{(U)} \frac{\lambda^{5} v v_{S_{1}} v_{\chi}}{2 \Lambda m_{\widetilde{T}}} & z_{12}^{(U)} y^{(U)} \frac{\lambda^{2} v v_{S_{1}} v_{\chi}}{2 \Lambda m_{\widetilde{T}}} & 0 \\
0 & z_{22}^{(U)} y^{(U)} \frac{\lambda v v_{S_{1}} v_{\chi}}{2 \Lambda m_{\widetilde{T}}} & 0 \\
0 & 0 & m_{t}
\end{array}\right) \\
& =\left(\begin{array}{ccc}
a_{11} \lambda^{8} & a_{12} \lambda^{5} & 0 \\
0 & a_{22} \lambda^{4} & 0 \\
0 & 0 & \alpha
\end{array}\right) \frac{v}{\sqrt{2}}, \\
& \tilde{M}_{D}=A_{D} M_{B}^{-1} B_{D}=\left(\begin{array}{ccc}
z_{11}^{(D)} \lambda^{5} & 0 & z_{13}^{(D)} \lambda^{4} \\
0 & z_{22}^{(D)} \lambda^{3} & z_{23}^{(D)} \lambda^{3} \\
0 & 0 & z_{3}^{(D)} \lambda
\end{array}\right) \frac{\lambda^{2} v_{S_{4}} v}{\sqrt{2} m_{B}} \\
& =\left(\begin{array}{ccc}
b_{11} \lambda^{7} & 0 & b_{13} \lambda^{6} \\
0 & b_{22} \lambda^{5} & b_{23} \lambda^{5} \\
0 & 0 & b_{33} \lambda^{3}
\end{array}\right) \frac{v}{\sqrt{2}}
\end{aligned}
$$

where we have set $\left(m_{B}\right)_{j}=m_{B}(j=1,2,3)$ and considered $m_{\widetilde{T}} \sim m_{B} \sim v_{\chi} \sim v_{S_{4}}$. Let us note that in our model, the dominant contribution to the Cabbibo mixing arises from the up-type quark sector, whereas the down-type quark sector contributes to the remaining CKM mixing angles. Given that we are considering real Yukawa couplings, in order to account for CP violation in the quark sector we take $v_{S_{3}}$ to be complex, which implies that the only complex entry in the SM quark mass matrices is $b_{13}$. Thus, in this scenario, and taking into account that the scalar $S_{3}$ is a $T^{\prime}$ doublet charged under the $Z_{12}$ symmetry as shown in Table 1 , the observed $\mathrm{CP}$ violation in the quark sector will arise from the spontaneous breaking of the $T^{\prime} \times Z_{12}$ discrete group by the vacuum expectation value of the $S_{3}$ scalar.
Table 5 Model and experimental values of the quark masses and CKM parameters

\begin{tabular}{lll}
\hline Observable & Model value & Experimental value \\
\hline$m_{u}(\mathrm{MeV})$ & 1.38 & $1.45_{-0.45}^{+0.56}$ \\
$m_{c}(\mathrm{MeV})$ & 635 & $635 \pm 86$ \\
$m_{t}(\mathrm{GeV})$ & 172.1 & $172.1 \pm 0.6 \pm 0.9$ \\
$m_{d}(\mathrm{MeV})$ & 2.9 & $2.9_{-0.4}^{+0.5}$ \\
$m_{s}(\mathrm{MeV})$ & 60.0 & $57.7_{-15.7}^{+16.8}$ \\
$m_{b}(\mathrm{GeV})$ & 2.82 & $2.82_{-0.04}^{+0.09}$ \\
$\sin \theta_{12}$ & 0.225 & 0.225 \\
$\sin \theta_{23}$ & 0.0412 & 0.0412 \\
$\sin \theta_{13}$ & 0.00365 & 0.00365 \\
$J$ & $3.30 \times 10^{-5}$ & $(3.18 \pm 0.15) \times 10^{-5}$ \\
\hline
\end{tabular}

The experimental values of the physical quark mass spectrum [195,196], mixing angles and Jarlskog invariant [197] can be obtained from the following benchmark point:

$$
\begin{aligned}
a_{11} \simeq 1.259, \quad a_{12} & \simeq-1.441, \quad a_{22} \\
& \simeq 1.400, \quad \alpha \simeq 0.989, \quad b_{11} \simeq 0.579 \\
b_{22} \simeq 0.604, \quad\left|b_{13}\right| & \simeq 1.265, \quad \arg \left(b_{13}\right) \\
& \simeq-158^{\circ}, \quad b_{23} \simeq 1.117, \quad b_{33} \simeq 1.431 .
\end{aligned}
$$

As indicated in Table 5 our model successfully reproduces the low energy quark flavor data by having the quark model parameters of order unity. The symmetries of our model give rise to quark mass matrix textures that successfully explain the SM quark mass spectrum and mixing parameters, without requiring the introduction of a hierarchy in the free effective parameters of the quark sector. These effective parameters only need to be mildly tuned in order to perfectly reproduce the observed quark mass spectrum and CKM parameters.

Finaly to close this section we briefly comment about the LHC signatures of exotic quarks in our model. As follows from the quark Yukawa terms of Eq. (2.6), the exotic quarks have mixing mass terms with all SM quarks, excepting the top quark. Such mixing terms allow that these exotic quarks can decay into any of the scalars of the model and a SM quark. These exotic quarks can decay into a SM quark and the SM-like Higgs boson. Such exotic quarks can be produced in pairs at the LHC via gluon fusion and Drell-Yan mechanism. Consequently, observing an excess of events in the six jet final state can be a signal of support of this model at the LHC. A detailed study of the exotic quark production at the LHC and the exotic quark decay modes is beyond the scope of this work and is left for future studies. 


\section{Lepton masses and mixings}

From the charged lepton Yukawa interactions given in Eq. (2.7) and using Eqs. (2.8) and (2.10) together with the product rules of the $T^{\prime}$ group shown in the "Appendix", we find that the charged lepton matrix is given by:

$$
\begin{aligned}
& M_{l}=\left(\begin{array}{ccc}
e^{-i \alpha} \cos \gamma & -e^{-i \alpha} \sin \gamma & 0 \\
e^{i \alpha} \sin \gamma & e^{i \alpha} \cos \gamma & 0 \\
0 & 0 & 1
\end{array}\right)
\end{aligned}
$$

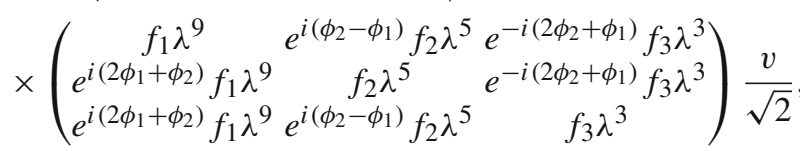

where $f_{i}$ with $i=1,2,3$ are $\mathcal{O}(1)$ dimensionless parameters assumed to be real.

Regarding the neutrino sector, from the lepton Yukawa terms given in Eq. (2.7), we find the following neutrino mass terms:

$-\mathcal{L}_{\text {mass }}^{(\nu)}=\frac{1}{2}\left(\overline{v_{L}^{C}} \overline{v_{R}} \overline{N_{R}}\right) M_{\nu}\left(\begin{array}{c}v_{L} \\ v_{R}^{C} \\ N_{R}^{C}\end{array}\right)+H . c$,

where the neutrino mass matrix $M_{\nu}$ is

$M_{v}=\left(\begin{array}{ccc}0_{3 \times 3} & M_{v_{D}} & 0_{3 \times 3} \\ M_{v_{D}}^{T} & 0_{3 \times 3} & M_{\chi} \\ 0_{3 \times 3} & M_{\chi}^{T} & M_{R}\end{array}\right)$,

and the submatrices $M_{\nu_{D}}$ and $M_{\chi}$ are generated from the $y_{\rho} \varepsilon_{a b c} \varepsilon_{d e c}\left(\bar{L}_{L}^{a}\left(L_{L}^{C}\right)^{b}\right)_{\mathbf{3}_{2}} \eta^{d} \chi^{e} \frac{\xi \sigma^{2}}{\Lambda^{4}}$ and $y_{\chi}^{(L)}\left(\bar{L}_{L} \chi N_{R}\right)_{\mathbf{1}}$ Yukawa terms in Eq. (2.7), respectively. Furthermore, the submatrix $M_{R}$ arises from the Majorana neutrino Yukawa interactions shown in the third line of Eq. (2.7). The submatrices $M_{v_{D}}, M_{\chi}$ and $M_{R}$ take the form:

$$
\begin{aligned}
M_{v_{D}} & =\left(\begin{array}{ccc}
0 & A e^{-i\left(2 \phi_{1}+\phi_{2}\right)} & -A e^{-i\left(2 \phi_{1}+\phi_{2}\right)} \\
-A e^{-i\left(2 \phi_{1}+\phi_{2}\right)} & 0 & A \\
A e^{-i\left(2 \phi_{1}+\phi_{2}\right)} & -A & 0
\end{array}\right), \\
M_{\chi} & =\left(\begin{array}{ccc}
B & 0 & 0 \\
0 & 0 & B e^{i\left(2 \phi_{1}+\phi_{2}\right)} \\
0 & B e^{i\left(2 \phi_{1}+\phi_{2}\right)} & 0
\end{array}\right), \\
M_{R} & =\left(\begin{array}{ccc}
(C+2 D) & -D e^{i\left(2 \phi_{1}+\phi_{2}\right)} & -D e^{i\left(2 \phi_{1}+\phi_{2}\right)} \\
-D e^{i\left(2 \phi_{1}+\phi_{2}\right)} & 2 D e^{3 i \phi_{1}} & (C-D) e^{i\left(2 \phi_{1}+\phi_{2}\right)} \\
-D e^{i\left(2 \phi_{1}+\phi_{2}\right)} & (C-D) e^{i\left(2 \phi_{1}+\phi_{2}\right)} & 2 D e^{3 i\left(\phi_{1}+\phi_{2}\right)}
\end{array}\right) .
\end{aligned}
$$

where $A, B, C$ and $D$ are given by:

$$
\begin{aligned}
& A=\frac{y_{\rho} v_{\eta} v_{\chi} v_{\xi} v_{\sigma}^{2}}{2 \sqrt{2} \Lambda^{4}}, \quad B=\frac{y_{\chi}^{(L)} v_{\chi}}{\sqrt{2}}, \\
& C=h_{1 N} \frac{v_{\sigma}^{6} v_{S_{6}}^{4}}{\Lambda^{10}} v_{\varphi}, \quad D=h_{2 N} \frac{v_{\xi} v_{\sigma}^{6} v_{S_{6}}^{4}}{\Lambda^{11}} v_{\varphi} .
\end{aligned}
$$

As shown in detail in Ref. [198], the full rotation matrix that diagonalizes the neutrino mass matrix $M_{\nu}$ is approximately given by

$\mathbb{U}=\left(\begin{array}{ccc}V_{v} & X_{3} U_{\chi} & X_{2} U_{R} \\ -\frac{\left(X_{2}^{\dagger}+X_{3}^{\dagger}\right)}{\sqrt{2}} V_{v} & \frac{(1-S)}{\sqrt{2}} U_{\chi} & \frac{(1+S)}{\sqrt{2}} U_{R} \\ -\frac{\left(X_{2}^{\dagger}-X_{3}^{\dagger}\right)}{\sqrt{2}} V_{v} & \frac{(-1-S)}{\sqrt{2}} U_{\chi} & \frac{(1-S)}{\sqrt{2}} U_{R}\end{array}\right)$,

where

$S=-\frac{1}{2 \sqrt{2} y_{\chi}^{(L)} v_{\chi}} M_{R}, \quad X_{2} \simeq X_{3} \simeq \frac{1}{y_{\chi}^{(L)} v_{\chi}} M_{v_{D}}^{*}$,

and the physical neutrino mass matrices are:

$M_{\nu}^{(1)}=M_{v_{D}}\left(M_{\chi}^{T}\right)^{-1} M_{R} M_{\chi}^{-1} M_{v_{D}}^{T}$,

$M_{\nu}^{(2)}=-\frac{1}{2}\left(M_{\chi}+M_{\chi}^{T}\right)+\frac{1}{2} M_{R}$,

$M_{v}^{(3)}=\frac{1}{2}\left(M_{\chi}+M_{\chi}^{T}\right)+\frac{1}{2} M_{R}$,

where $M_{\nu}^{(1)}$ is the light active neutrino mass matrix whereas $M_{v}^{(2)}$ and $M_{v}^{(3)}$ are the exotic Dirac neutrino mass matrices. The physical neutrino spectrum is composed of 3 light active neutrinos and 6 nearly degenerate sterile exotic pseudo-Dirac neutrinos.

Furthermore, from Eqs. (4.4)-(4.7) and (4.10), we find for the light active neutrino mass scale, the estimate $m_{v} \sim$ $\lambda^{22} v_{\varphi} \sim 50 \mathrm{meV}$. Consequently, our model provides a natural explanation for the smallness of the light active neutrino masses.

The sterile neutrinos can be pair produced at the Large Hadron Collider (LHC), via a Drell-Yan annihilation mediated by a heavy $Z^{\prime}$ gauge boson. The sterile neutrinos mix the light active ones thus allowing the sterile neutrinos to decay into SM particles, so that the final decay products will be a SM charged lepton and a $W$ gauge boson. Consequently, the observation of an excess of events in the dilepton final states above the SM background, can be a signal in support of this model at the LHC. Studies of inverse seesaw neutrino signatures at the colliders as well as the production of heavy neutrinos at the LHC are carried out in Refs. [199-211]. A comprehensive study of the implications of our model at colliders goes beyond the scope of this work and will be done elsewhere.

By varying the lepton sector model parameters, we obtain values for the charged lepton masses, neutrino mass squared differences and leptonic mixing parameters in very good agreement with the experimental data, as shown in Table 6 . This shows that our model can successfully accommodate 
Table 6 The model values shown in the table are the best fit results for the neutrino mass squared differences, mixing angles and the $\mathrm{CP}$-violating phase for the case of normal hierarchy. The $1-3 \sigma$ experimental ranges [212] are also shown for comparison

\begin{tabular}{lllll}
\hline Observable & Model value & \multicolumn{2}{l}{ Experimental value } & \\
\cline { 2 - 4 } & & $1 \sigma$ range & $2 \sigma$ range & $3 \sigma$ range \\
\hline$m_{e}[\mathrm{MeV}]$ & 0.487 & 0.487 & 0.487 & 0.487 \\
$m_{\mu}[\mathrm{MeV}]$ & 102.8 & $102.8 \pm 0.0003$ & $102.8 \pm 0.0006$ & $102.8 \pm 0.0009$ \\
$m_{\tau}[\mathrm{GeV}]$ & 1.75 & $1.75 \pm 0.0003$ & $1.75 \pm 0.0006$ & $1.75 \pm 0.0009$ \\
$\Delta m_{21}^{2}\left[10^{-5} e V^{2}\right]$ & 7.54987 & $7.55_{-0.16}^{+0.20}$ & $7.20-7.94$ & $7.05-8.14$ \\
$\Delta m_{31}^{2}\left[10^{-3} e V^{2}\right]$ & 2.49995 & $2.50 \pm 0.03$ & $2.44-2.57$ & $2.41-2.60$ \\
$\sin ^{2}\left(\theta_{12}\right) / 10^{-1}$ & 3.19999 & $3.20_{-0.16}^{+0.20}$ & $2.89-3.59$ & $2.73-3.79$ \\
$\sin ^{2}\left(\theta_{23}\right) / 10^{-1}$ & 4.91197 & $5.47_{-0.30}^{+0.20}$ & $4.67-5.83$ & $4.45-5.99$ \\
$\sin ^{2}\left(\theta_{13}\right) / 10^{-2}$ & 2.16073 & $2.160_{-0.069}^{+0.083}$ & $2.03-2.34$ & $1.96-2.41$ \\
$\delta_{C P}$ & $192.761^{\circ}$ & $218_{-27^{\circ}}^{+38^{\circ}}$ & $182^{\circ}-315^{\circ}$ & $157^{\circ}-349^{\circ}$
\end{tabular}

the experimental values of the physical observables of the lepton sector. It is worth mentioning that the range for the experimental values in Table 6 were taken from [212] for the case of normal hierarchy. Let us note that we only consider the case of normal hierarchy since it is favored over more than $3 \sigma$ than the inverted neutrino mass ordering. Furthermore, let us note that given that we are considering real Yukawa couplings in our model, the observed CP violation in the lepton sector is generated by the spontaneous breaking of the $T^{\prime} \times Z_{6} \times Z_{8}$ discrete group by the vacuum expectation values of the $\rho, \phi, \zeta$ and $\xi$ scalars.

Figure 1 shows the correlation between the leptonic mixing parameters and the leptonic Dirac CP violating phase for the case of normal neutrino mass hierarchy. To obtain these figures, the lepton sector parameters were randomly generated in a range of values where the neutrino mass squared splittings and leptonic mixing parameters are inside the $3 \sigma$ experimentally allowed range. We found a leptonic Dirac CP violating phase in the range $180^{\circ} \lesssim \delta_{C P} \lesssim 205^{\circ}$, whereas the leptonic mixing parameters are obtained to be in the ranges $0.3196 \lesssim \sin ^{2} \theta_{12} \lesssim 0.3202,0.4900 \lesssim \sin ^{2} \theta_{23} \lesssim$ 0.4925 and $0.0205 \lesssim \sin ^{2} \theta_{13} \lesssim 0.0240$.

\section{Higgs diphoton rate}

The explicit form of the $h \rightarrow \gamma \gamma$ decay rate is [213-218]

$$
\begin{aligned}
& \Gamma(h \rightarrow \gamma \gamma)=\frac{\alpha_{e m}^{2} m_{h}^{3}}{256 \pi^{3} v^{2}} \\
& \left|\sum_{f} a_{h f f} N_{C} Q_{f}^{2} F_{1 / 2}\left(\rho_{f}\right)+a_{h W W} F_{1}\left(\rho_{W}\right)+a_{h W^{\prime} W^{\prime}} F_{1}\left(\rho_{W^{\prime}}\right)\right|^{2} .
\end{aligned}
$$

Here $\rho_{i}$ are the mass ratios $\rho_{i}=\frac{m_{h}^{2}}{4 M_{i}^{2}}$ with $M_{i}=$ $m_{f}, M_{W}, M_{W^{\prime}} ; \alpha_{e m}$ is the fine structure constant; $N_{C}$ is the color factor $\left(N_{C}=1\right.$ for leptons and $N_{C}=3$ for quarks); and $Q_{f}$ is the electric charge of the fermion in the loop.

From the fermionic loop contributions, we only consider the one arising from the top quark exchange. Furthermore, $a_{h t t}, a_{h W W}$ and $a_{h W^{\prime} W^{\prime}}$ are the deviation factors from the SM expectation, of the Higgs-top quark coupling, the HiggsWW and the Higgs-W'W' gauge boson couplings, respectively:

$$
\begin{aligned}
a_{h W W} & =-\sin \alpha, \\
a_{h W^{\prime} W^{\prime}} & =\cos \alpha \cot \beta, \\
a_{h t t} & \simeq 1 .
\end{aligned}
$$

The numerical values of these parameters are given in Table 7. Let us note that in our model the Higgs-top quark coupling is very close to the $\mathrm{SM}$ expectation, i.e., $a_{h t t} \simeq 1$, since the mixing between the CP even neutral scalar fields $\xi_{\eta}$ and $\xi_{\chi}$ is very suppressed, being the $126 \mathrm{GeV} \mathrm{SM} \mathrm{like} \mathrm{Higgs} \mathrm{boson}$ mainly composed of the $\xi_{\eta}$ field.

The dimensionless loop factors $F_{1 / 2}(\rho)$ and $F_{1}(\rho)$ for spin-1/2 and spin-1 particles in the loop, respectively are [213-220]:

$$
\begin{aligned}
F_{1 / 2}(\rho) & =2(\rho+(\rho-1) f(\rho)) \rho^{-2}, \\
F_{1}(\rho) & =-2\left(2 \rho^{2}+3 \rho+3(2 \rho-1) f(\rho)\right) \rho^{-2}, \\
F_{0} & =-(\rho-f(\rho)) \rho^{-2},
\end{aligned}
$$

with

$f(\rho)= \begin{cases}\arcsin ^{2} \sqrt{2} & \text { for } \rho \leq 1 \\ -\frac{1}{4}\left(\ln \left(\frac{1+\sqrt{1-\rho^{-1}}}{1-\sqrt{1-\rho^{-1}}-i \pi}\right)^{2}\right) & \text { for } \rho>1 .\end{cases}$

In what follows we show that our model is consistent with the current Higgs diphoton decay rate constraints. To this end, we introduce the ratio $R_{\gamma \gamma}$, which normalizes the $\gamma \gamma$ signal 


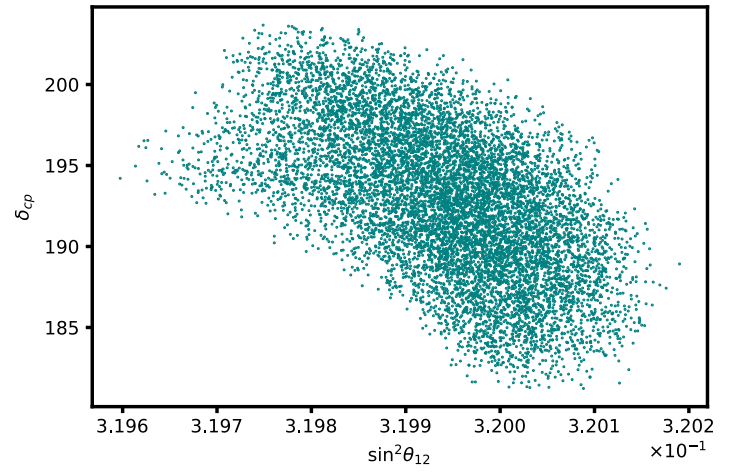

(a) Correlation between the solar mixing parameter $\sin ^{2} \theta_{12}$ and the leptonic Dirac CP-violating phase $\delta_{C P}$.

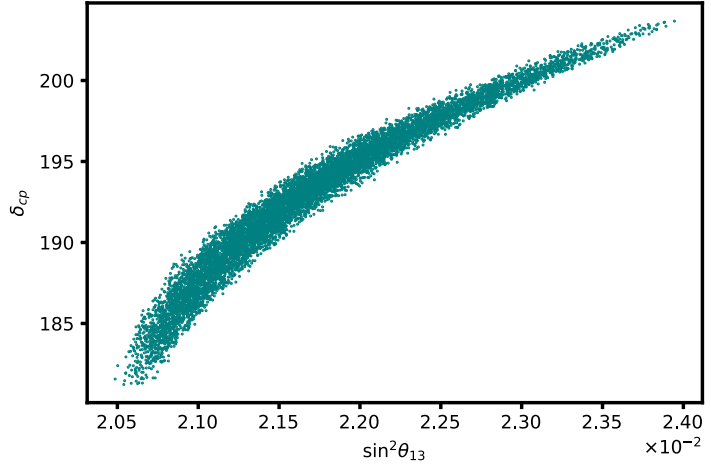

(b) Correlation between the reactor mixing parameter $\sin ^{2} \theta_{13}$ and the leptonic Dirac CP-violating phase $\delta_{C P}$.

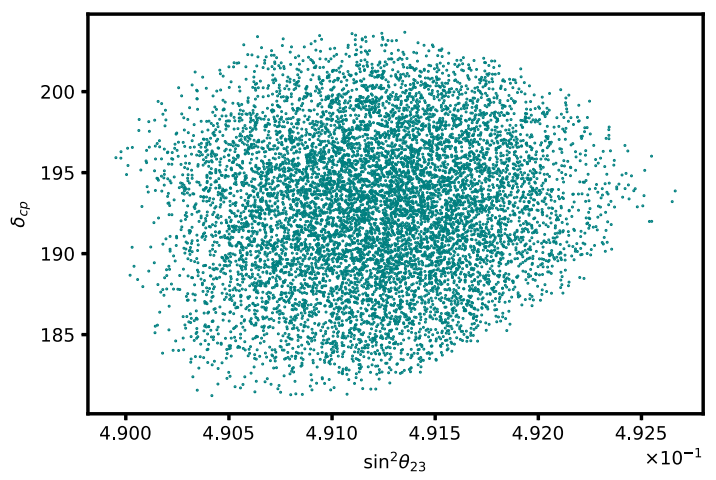

(c) Correlation between the atmospheric mixing parameter $\sin ^{2} \theta_{23}$ and the leptonic Dirac CP-violating phase $\delta_{C P}$.

Fig. 1 Correlations between the leptonic mixing parameters and the leptonic Dirac CP violating phase

predicted by our model relative to that of the SM:

$R_{\gamma \gamma}=\frac{\sigma(p p \rightarrow h) \Gamma(h \rightarrow \gamma \gamma)}{\sigma(p p \rightarrow h)_{S M} \Gamma\left(h \rightarrow \gamma \gamma_{S M}\right.} \simeq a_{h t t}^{2} \frac{\Gamma(h \rightarrow \gamma \gamma)}{\Gamma(h \rightarrow \gamma \gamma)_{S M}}$.

The normalization given by (5.9) for $h \rightarrow \gamma \gamma$ was also used in [140,218, 221-225].

The ratio $R_{\gamma \gamma}$ has been measured by CMS and ATLAS with the best fit signals [226,227]:

$R_{\gamma \gamma}^{C M S}=1.14_{-0.23}^{+0.26}$ and $R_{\gamma \gamma}^{A T L A S}=1.17 \pm 0.27$

With the best fit results shown in Table 7 the $R_{\gamma \gamma}$ parameter has been calculated as:

$R_{\gamma \gamma}=1.0021$

Consequently, our model successfully accommodates the current Higgs diphoton decay rate constraints.
Table 7 Numerical values for the deviation factors $a_{h t t}, a_{h W W}$ and $a_{h W^{\prime} W^{\prime}}$ used for the computation of the Higgs diphoton decay rate. Here we set $v_{\chi}=10 \mathrm{TeV}$

\begin{tabular}{ll}
\hline Parameters & Model value \\
\hline$a_{h W^{-} W^{+}}$ & 0.999816 \\
$a_{h W^{\prime} W^{\prime}}$ & 0.000471445 \\
$a_{h t t}$ & 1.0 \\
\hline
\end{tabular}

\section{Lepton flavor violating constraints}

In this section we will determine the constraints on the model parameter space imposed by the charged lepton flavor violating processes $\mu \rightarrow e \gamma, \tau \rightarrow \mu \gamma$ and $\tau \rightarrow e \gamma$. As mentioned in the previous section, the sterile neutrino spectrum of the model is composed of six nearly degerate heavy neutrinos. These sterile neutrinos together with the heavy $W^{\prime}$ gauge boson induce the $l_{i} \rightarrow l_{j} \gamma$ decay at one loop level, whose Branching ratio is given by: $[2,228,229]$ :

$B r\left(l_{i} \rightarrow l_{j} \gamma\right)=\frac{\alpha_{W}^{3} s_{W}^{2} m_{l_{i}}^{5}}{256 \pi^{2} m_{W^{\prime}}^{4} \Gamma_{i}}\left|G\left(\frac{m_{N}^{2}}{m_{W^{\prime}}^{2}}\right)\right|^{2}$, 
$G(x)=-\frac{2 x^{3}+5 x^{2}-x}{4(1-x)^{2}}-\frac{3 x^{3}}{2(1-x)^{4}} \ln x$.

where the one loop level contribution arising from the $W$ gauge boson exchange has been neglected because it is suppressed by the quartic power of the active-sterile neutrino mixing angle $\theta$, which in our model is of the order of $10^{-3}$, for sterile neutrino masses of about $1 \mathrm{TeV}$. It has been shown in Ref. [230], that for such mixing angle the contribution of the $W$ gauge boson to the branching ratio for the $\mu \rightarrow e \gamma$ decay rate takes values of the order of $10^{-16}$, which corresponds to three orders of magnitude below its experimental upper limit of $4.2 \times 10^{-13}$. Thus, in this work, we only consider the dominant $W^{\prime}$ contribution to the $\mu \rightarrow e \gamma$ decay rate.

Figure 2 shows the allowed parameter space in the $m_{W^{\prime}}-m_{N}$ plane consistent with the constraints arising from charged lepton flavor violating decays. The $W^{\prime}$ gauge boson and the sterile neutrino masses have been taken to be in the ranges $4 \mathrm{TeV} \lesssim m_{W^{\prime}} \lesssim 8 \mathrm{TeV}$ and $1 \mathrm{TeV} \lesssim m_{N} \lesssim 4.5$ $\mathrm{TeV}$, respectively. Notice that we have considered $W^{\prime}$ gauge boson masses larger than $4 \mathrm{TeV}$ to fulfill the constraints arising from on $K, D$ and $B$ meson mixings [185]. As seen from Fig. 2, the obtained values for the branching ratio of $\mu \rightarrow e \gamma$ decay are below its experimental upper limit of $4.2 \times 10^{-13}$ and are within the reach of future experimental sensitivity, in the allowed model parameter space. In the region of parameter space consistent with $\mu \rightarrow e \gamma$ decay rate constraints, the maximum obtained branching ratios for the $\tau \rightarrow \mu \gamma$ and $\tau \rightarrow e \gamma$ decays can reach values of the order of $10^{-13}$, which is four orders of magnitude below their corresponding upper experimental bounds of $4.4 \times 10^{-8}$ and $3.3 \times 10^{-8}$, respectively. Consequently, our model is compatible with the charged lepton flavor violating decay constaints provided that the sterile neutrino are lighter than about 1.6 $\mathrm{TeV}$ and $4.5 \mathrm{TeV}$ for $W^{\prime}$ gauge boson masses of $4 \mathrm{TeV}$ and $8 \mathrm{TeV}$, respectively.

Finally, to close this section we provide some comments regarding the decays of quasi-Dirac sterile neutrinos as well as a comparison of our predictions for such decays and for the LFV signals with the ones obtained in other models with extended gauge symmetry. Notice that, as in the $U^{\prime}$ (1) model of Ref. [230], in our model the sterile neutrinos feature the two body decay modes: $N \rightarrow l_{i}^{ \pm} W^{\mp}$, $v_{i} Z$ and $v_{i} h$ (where $i=1,2,3$ is a flavor index), which are suppressed by the small active-sterile neutrino mixing angle $\theta \sim \mathcal{O}\left(10^{-3}\right)$. Those two body decay modes, give rise to the three body decay modes for the sterile neutrinos: $N \rightarrow l_{i}^{+} l_{j}^{-} v_{k}, N \rightarrow l_{i}^{-} u_{j} \bar{d}_{k}, N \rightarrow b \bar{b} v_{k}$ (where $i, j, k=1,2,3$ are flavor indices). Such aforementioned decay modes for the sterile neutrinos are also presented in the $U^{\prime}$ (1) model of Ref. [230]. Consequently, we expect similar predictions for the total cross section of the LFV signal

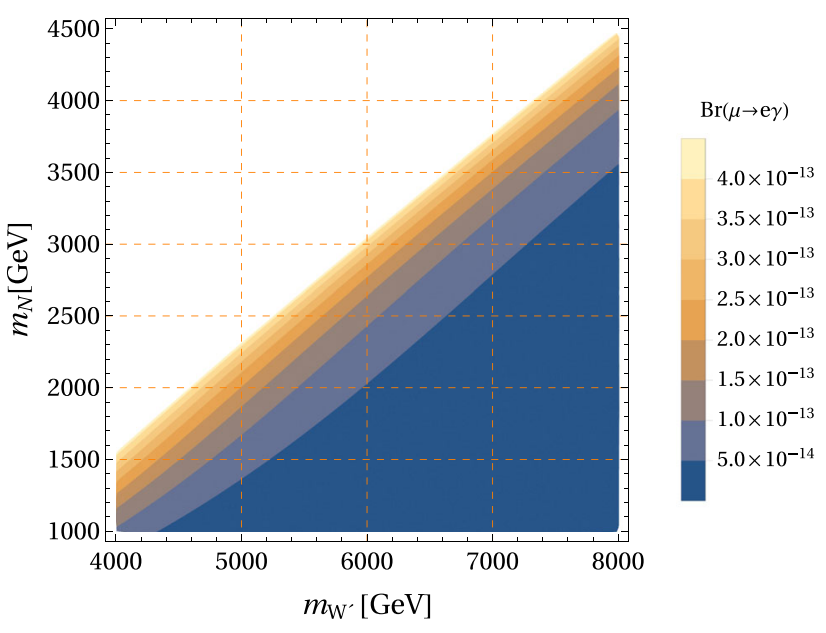

Fig. 2 Allowed parameter space in the $m_{W^{\prime}}-m_{N}$ plane consistent with the LFV constraints

process $p p \rightarrow N N \rightarrow e^{ \pm} \mu^{\mp} 4 j$ as well as for the sterile neutrino decays, to the ones obtained in Ref. [230]. A slightly different prediction is expected for the $N \rightarrow l_{i}^{+} l_{j}^{-} v_{k}$ decay rate, which in our model receives contributions from the diagrams involving the off-shell $W$ and $W^{\prime}$ gauge bosons, whereas in the model of Ref. [230], it only receives contribution from the diagram involving the off-shell $W$ gauge boson. It is worth mentioning that the contribution to the $N \rightarrow l_{i}^{+} l_{j}^{-} v_{k}$ decay rate arising from the the exchange of the off-shell $W^{\prime}$ gauge bosons is strongly suppressed by a factor of about $\frac{M_{W}^{4}}{M_{W^{\prime}}^{4}}$ when compared with the one due to the $W$ exchange. Despite such similar expectations for the aforementioned LFV signals, it is worth mentioning that for an active-sterile neutrino mixing angle $\theta \sim \mathcal{O}\left(10^{-3}\right)$, our obtained values for the branching ratio of the $\mu \rightarrow e \gamma$ decay rate will be about three orders of magnitude larger than the obtained in Ref. [230]. This is due to the fact that in our model the $\mu \rightarrow e \gamma$ decay is dominated by the $W^{\prime}$ contribution (absent in the $U^{\prime}$ (1) model of Ref. [230]), which is much larger than the contribution arising from the $W$ exchange. Furthermore, we expect similar predictions for the sterile neutrino decay rates and for the cross section of the LFV signal process $p p \rightarrow N N \rightarrow e^{ \pm} \mu^{\mp}$ to the ones obtained in the left-right symmetric model of Refs. [231,232]. Despite such similar predictions, the charged lepton flavor violating process $\mu \rightarrow e \gamma$ can be used to discriminate our model from the left-right symmetric model of Refs. [231,232], where in the former its dominant contribution arises from the loop diagram involving the $W^{\prime}$ gauge boson exchange, whereas in the latter it receives contributions from the exchange of the $W_{R}$ gauge boson and the doubly charged scalars contained in the $S U(2)_{L}$ and $S U(2)_{R}$ scalar triplets. 


\section{Conclusions}

We have constructed a viable 3-3-1 model with two $S U(3)_{L}$ scalar triplets, extended fermion and scalar spectrum, based on the $T^{\prime}$ family symmetry and other auxiliary cyclic symmetries, whose spontaneous breaking produces the observed pattern of SM fermion masses and mixing angles. In our model the SM quarks lighter than the top quark, get their masses from a low scale Universal seesaw mechanism, whereas the SM charged lepton masses are produced by a Froggatt-Nielsen mechanism. In addition, the small light active neutrino masses are generated from an inverse seesaw mechanism. Our model is consistent with the low energy SM fermion flavor data and successfully accommodates the current Higgs diphoton decay rate constraints as well as the constraints arising from charged lepton flavor violating processes. In particular, we have found that the constraint on the charged lepton flavor violating decay $\mu \rightarrow e \gamma$ sets the sterile neutrino masses to be lighter than about $1.6 \mathrm{TeV}$ and $4.5 \mathrm{TeV}$ for $W^{\prime}$ gauge boson masses of $4 \mathrm{TeV}$ and $8 \mathrm{TeV}$, respectively. We have found that in the allowed region of parameter space, the obtained maximum values of the $\mu \rightarrow e \gamma$ branching ratio are close to about $4 \times 10^{-13}$, which is within the reach of future experimental sensitivity. Furthermore, the obtained branching ratios for the $\tau \rightarrow \mu \gamma$ and $\tau \rightarrow e \gamma$ decays can reach values of the order of $10^{-13}$. Consequently, our model predicts charged lepton flavor violating decays within the reach of future experimental sensitivity.

Acknowledgements This research has received funding from Fondecyt (Chile), Grants No. 1170803, CONICYT PIA/Basal FB0821, and Programa de Incentivos a la Iniciación Científica (PIIC) from UTFSM (Chile).

Data Availability Statement This manuscript has no associated data or the data will not be deposited. [Authors' comment: Given that our work is theoretical, we did not employ experimental data in our numerical computations that give rise to Figs. 1 and 2, Table 7 and second column of Tables 5 and 6. Furthermore, the plots in Figs. 1 and 2 correspond to the correlations between the leptonic mixing parameters and the leptonic Dirac CP violating phase and the allowed model parameter space consistent with the LFV constraints and its prediction obtained in our model. The only experimental data used in our work are the ones reported in the third column of Tables 5 and 6 and the values given by Eq. (5.10).]

Open Access This article is distributed under the terms of the Creative Commons Attribution 4.0 International License (http://creativecomm ons.org/licenses/by/4.0/), which permits unrestricted use, distribution, and reproduction in any medium, provided you give appropriate credit to the original author(s) and the source, provide a link to the Creative Commons license, and indicate if changes were made.

Funded by SCOAP ${ }^{3}$.

\section{Appendix A: The product rules for T'}

The double tetrahedral group $T^{\prime}$ is the smallest discrete subgroup of $S U(2)$ as well as the smallest group of any kind with 1-, 2- and 3-dimensional representations and the multiplication rule $\mathbf{2} \otimes \mathbf{2}=\mathbf{3} \oplus \mathbf{1}$, thus allowing to reproduce the successful $U(2)$ textures [66]. It has the following tensor product rules [233]:

$$
\begin{aligned}
& \left(\begin{array}{l}
x_{1} \\
x_{2}
\end{array}\right)_{\mathbf{2}\left(\mathbf{2}^{\prime}\right)} \otimes\left(\begin{array}{l}
y_{1} \\
y_{2}
\end{array}\right)_{\mathbf{2}\left(\mathbf{2}^{\prime \prime}\right)}^{3]:}=\left(\frac{x_{1} y_{2}-x_{2} y_{1}}{\sqrt{2}}\right)_{\mathbf{1}} \\
& \oplus\left(\begin{array}{c}
\frac{i}{\sqrt{2}} p_{1} p_{2} \bar{p}\left(x_{1} y_{2}+x_{2} y_{1}\right) \\
p_{2} \bar{p}^{2} x_{1} y_{1} \\
x_{2} y_{2}
\end{array}\right)_{3} \\
& \left(\begin{array}{l}
x_{1} \\
x_{2}
\end{array}\right)_{\mathbf{2}^{\prime}(\mathbf{2})} \otimes\left(\begin{array}{l}
y_{1} \\
y_{2}
\end{array}\right)_{\mathbf{2}^{\prime}\left(\mathbf{2}^{\prime \prime}\right)}=\left(\frac{x_{1} y_{2}-x_{2} y_{1}}{\sqrt{2}}\right)_{\mathbf{1}^{\prime \prime}} \\
& \oplus\left(\begin{array}{c}
p_{1} \bar{p}^{2} x_{1} y_{1} \\
x_{2} y_{2} \\
\frac{i}{\sqrt{2}} \bar{p} \bar{p}_{2}\left(x_{1} y_{2}+x_{2} y_{1}\right)
\end{array}\right)_{\mathbf{3}}, \\
& \left(\begin{array}{l}
x_{1} \\
x_{2}
\end{array}\right)_{\mathbf{2}^{\prime \prime}(\mathbf{2})} \otimes\left(\begin{array}{l}
y_{1} \\
y_{2}
\end{array}\right)_{\mathbf{2}^{\prime \prime}\left(\mathbf{2}^{\prime}\right)}=\left(\frac{x_{1} y_{2}-x_{2} y_{1}}{\sqrt{2}}\right)_{\mathbf{1}^{\prime}} \\
& \oplus\left(\begin{array}{c}
x_{2} y_{2} \\
\frac{i}{\sqrt{2}} \bar{p}_{1}\left(x_{1} y_{2}+x_{2} y_{1}\right) \\
\bar{p}^{2} \bar{p}_{1} \bar{p}_{2} x_{1} y_{1}
\end{array}\right)_{\mathbf{3}} . \\
& 2 \times 2^{\prime}=2^{\prime \prime} \times 2^{\prime \prime}, \quad 2 \times 2^{\prime \prime}=2^{\prime} \times 2^{\prime}, \\
& 2^{\prime} \times 2^{\prime \prime}=2 \times 2, \\
& \left(\begin{array}{l}
x_{1} \\
x_{2} \\
x_{3}
\end{array}\right)_{\mathbf{3}} \otimes\left(\begin{array}{l}
y_{1} \\
y_{2} \\
y_{3}
\end{array}\right)_{\mathbf{3}}=\left[x_{1} y_{1}+p_{1}^{2} p_{2}\left(x_{2} y_{3}+x_{3} y_{2}\right)\right]_{\mathbf{1}} \\
& \oplus\left[x_{3} y_{3}+\bar{p}_{1} \bar{p}_{2}^{2}\left(x_{1} y_{2}+x_{2} y_{1}\right)\right]_{\mathbf{1}^{\prime}} \\
& \oplus\left[\left(x_{2} y_{2}+\bar{p}_{1} p_{2}\left(x_{1} y_{3}+x_{3} y_{1}\right)\right]_{\mathbf{1}^{\prime \prime}}\right. \\
& \oplus\left(\begin{array}{c}
2 x_{1} y_{1}-p_{1}^{2} p_{2}\left(x_{2} y_{3}+x_{3} y_{3}\right) \\
2 p_{1} p_{2}^{2} x_{3} y_{3}-x_{1} y_{2}-x_{2} y_{1} \\
2 p_{1} \bar{p}_{2} x_{2} y_{2}-x_{1} y_{3}-x_{3} y_{1}
\end{array}\right)_{\mathbf{3}_{\mathbf{1}}} \\
& \oplus\left(\begin{array}{c}
x_{2} y_{3}-x_{3} y_{2} \\
\bar{p}_{1}^{2} \bar{p}_{2}\left(x_{1} y_{2}-x_{2} y_{1}\right) \\
\bar{p}_{1}^{2} \bar{p}_{2}\left(x_{3} y_{1}-x_{1} y_{3}\right)
\end{array}\right)_{\mathbf{3}_{2}},
\end{aligned}
$$

where $p_{1}=e^{i \phi_{1}}$ and $p_{2}=e^{i \phi_{2}}$.

\section{Appendix B: Scalar potential for one of the $T^{\prime}$ scalar} triplets

The scalar potential for the $T^{\prime}$ scalar triplet $\rho$ is given by:

$$
\begin{aligned}
V= & -\mu_{\rho}^{2}\left(\rho \rho^{*}\right)_{\mathbf{1}}+\kappa_{1}\left(\rho \rho^{*}\right) \mathbf{1}^{\prime}\left(\rho \rho^{*}\right)_{\mathbf{1}^{\prime \prime}}+\kappa_{2}\left(\rho \rho^{*}\right) \mathbf{1}_{\mathbf{1}^{\prime \prime}}\left(\rho \rho^{*}\right)_{\mathbf{1}^{\prime}} \\
& +\kappa_{3}\left(\rho \rho^{*}\right)_{\mathbf{3}_{1}}\left(\rho \rho^{*}\right) \mathbf{3}_{1}+\kappa_{4}\left(\rho \rho^{*}\right) \mathbf{3}_{2}\left(\rho \rho^{*}\right) \mathbf{3}_{2} \\
& +\kappa_{5}\left(\rho \rho^{*}\right)_{\mathbf{3}_{1}}\left(\rho \rho^{*}\right) \mathbf{3}_{2}+\text { h.c. }
\end{aligned}
$$

This scalar potential has six free parameters: one bilinear and five quartic couplings. The $\mu_{\rho}$ parameter can be writ- 
ten as a function of the other five parameters by the scalar potential minimization condition:

$$
\begin{aligned}
& \frac{\partial\langle V(\rho)\rangle}{\partial v_{\rho}}=8\left(\cos (\gamma)-e^{-i\left(2 \phi_{1}+\phi_{2}\right)} \sin (\gamma)\right)^{2} \\
& \times\left(\cos (\gamma)-e^{i\left(2 \phi_{1}+\phi_{2}\right)} \sin (\gamma)\right)^{2} \kappa_{1} v_{\rho}^{3} \\
& +8\left(\cos (\gamma)-e^{-i\left(2 \phi_{1}+\phi_{2}\right)} \sin (\gamma)\right)^{2} \\
& \times\left(\cos (\gamma)-e^{i\left(2 \phi_{1}+\phi_{2}\right)} \sin (\gamma)\right)^{2} \kappa_{2} v_{\rho}^{3} \\
& +32\left(\cos (\gamma)-e^{-i\left(2 \phi_{1}+\phi_{2}\right)} \sin (\gamma)\right)^{2} \\
& \left(\cos (\gamma)-e^{i\left(2 \phi_{1}+\phi_{2}\right)} \sin (\gamma)\right)^{2} \kappa_{3} v_{\rho}^{3} \\
& +2\left(\cos (\gamma)-e^{-i\left(2 \phi_{1}+\phi_{2}\right)} \sin (\gamma)\right) \\
& \times\left(\cos (\gamma)-e^{i\left(2 \phi_{1}+\phi_{2}\right)} \sin (\gamma)\right) \\
& \times \kappa_{5}\left(2 e^{i \alpha}\left(\cos (\gamma)-e^{-i\left(2 \phi_{1}+\phi_{2}\right)} \sin (\gamma)\right) v_{\rho}\right. \\
& \left.-2 e^{-i \alpha}\left(\cos (\gamma)-e^{i\left(2 \phi_{1}+\phi_{2}\right)} \sin (\gamma)\right) v_{\rho}\right) v_{\rho}^{2} \\
& +2\left(\cos (\gamma)-e^{-i\left(2 \phi_{1}+\phi_{2}\right)} \sin (\gamma)\right) \\
& \times\left(\cos (\gamma)-e^{i\left(2 \phi_{1}+\phi_{2}\right)} \sin (\gamma)\right) \\
& \times \kappa_{5}\left(2 e^{-i \alpha}\left(\cos (\gamma)-e^{i\left(2 \phi_{1}+\phi_{2}\right)} \sin (\gamma)\right) v_{\rho}\right. \\
& \left.-2 e^{i \alpha}\left(\cos (\gamma)-e^{-i\left(2 \phi_{1}+\phi_{2}\right)} \sin (\gamma)\right) v_{\rho}\right) v_{\rho}^{2} \\
& -4\left(\cos (\gamma)-e^{-i\left(2 \phi_{1}+\phi_{2}\right)} \sin (\gamma)\right) \\
& \times\left(\cos (\gamma)-e^{i\left(2 \phi_{1}+\phi_{2}\right)} \sin (\gamma)\right) \mu_{\rho}^{2} v_{\rho} \\
& +4\left(\cos (\gamma)-e^{-i\left(2 \phi_{1}+\phi_{2}\right)} \sin (\gamma)\right) \\
& \times\left(\cos (\gamma)-e^{i\left(2 \phi_{1}+\phi_{2}\right)} \sin (\gamma)\right) \\
& \times \kappa_{5}\left(e^{i \alpha}\left(\cos (\gamma)-e^{-i\left(2 \phi_{1}+\phi_{2}\right)} \sin (\gamma)\right) v_{\rho}^{2}-e^{-i \alpha}\right. \\
& \left.\times\left(\cos (\gamma)-e^{i\left(2 \phi_{1}+\phi_{2}\right)} \sin (\gamma)\right) v_{\rho}^{2}\right) v_{\rho} \\
& +4\left(\cos (\gamma)-e^{-i\left(2 \phi_{1}+\phi_{2}\right)} \sin (\gamma)\right) \\
& \times\left(\cos (\gamma)-e^{i\left(2 \phi_{1}+\phi_{2}\right)} \sin (\gamma)\right) \\
& \times \kappa_{5}\left(e^{-i \alpha}\left(\cos (\gamma)-e^{i\left(2 \phi_{1}+\phi_{2}\right)} \sin (\gamma)\right) v_{\rho}^{2}-e^{i \alpha}\right. \\
& \left.\times\left(\cos (\gamma)-e^{-i\left(2 \phi_{1}+\phi_{2}\right)} \sin (\gamma)\right) v_{\rho}^{2}\right) v_{\rho} \\
& +2 \kappa_{4}\left(2 e^{i \alpha}\left(\cos (\gamma)-e^{-i\left(2 \phi_{1}+\phi_{2}\right)} \sin (\gamma)\right) v_{\rho}-2 e^{-i \alpha}\right. \\
& \left.\times\left(\cos (\gamma)-e^{i\left(2 \phi_{1}+\phi_{2}\right)} \sin (\gamma)\right) v_{\rho}\right) \\
& \times\left(e^{i \alpha}\left(\cos (\gamma)-e^{-i\left(2 \phi_{1}+\phi_{2}\right)} \sin (\gamma)\right) v_{\rho}^{2}-e^{-i \alpha}\right. \\
& \left.\times\left(\cos (\gamma)-e^{i\left(2 \phi_{1}+\phi_{2}\right)} \sin (\gamma)\right) v_{\rho}^{2}\right) \\
& +2 \kappa_{4}\left(2 e^{-i \alpha}\left(\cos (\gamma)-e^{i\left(2 \phi_{1}+\phi_{2}\right)} \sin (\gamma)\right) v_{\rho}-2 e^{i \alpha}\right. \\
& \left.\times\left(\cos (\gamma)-e^{-i\left(2 \phi_{1}+\phi_{2}\right)} \sin (\gamma)\right) v_{\rho}\right)
\end{aligned}
$$

$$
\begin{aligned}
& \times\left(e^{-i \alpha}\left(\cos (\gamma)-e^{i\left(2 \phi_{1}+\phi_{2}\right)} \sin (\gamma)\right) v_{\rho}^{2}-e^{i \alpha}\right. \\
& \left.\times\left(\cos (\gamma)-e^{-i\left(2 \phi_{1}+\phi_{2}\right)} \sin (\gamma)\right) v_{\rho}^{2}\right) \\
= & 0
\end{aligned}
$$

Here for the sake of simplicity we consider vanishing phases in the multiplications rules for the tensor product of the scalar triplets of $T^{\prime}$. Then, the scalar potential minimization condition yields the following relation:

$$
\begin{aligned}
\mu_{\rho}^{2}= & v_{\rho}^{2} \\
& \times\left(\frac{16 \kappa_{4}\left(\sin (\alpha) \cos (\gamma)-\sin (\gamma) \sin \left(\alpha-2 \phi_{1}-\phi_{2}\right)\right)^{2}}{\sin \left(2 \gamma-2 \phi_{1}-\phi_{2}\right)+\sin \left(2\left(\gamma+\phi_{1}\right)+\phi_{2}\right)-2}\right. \\
& \left.-2\left(\kappa_{1}+\kappa_{2}+4 \kappa_{3}\right)\left(\sin (2 \gamma) \cos \left(2 \phi_{1}+\phi_{2}\right)-1\right)\right)(\mathrm{B} .3)
\end{aligned}
$$

This result indicates that the VEV pattern of the $T^{\prime}$ triplet $\rho$ in (2.8) is consistent with a global minimum of the scalar potential (B.1) of this model for a large region of parameter space. Following the same procedure previously described, one can also show that the VEV patterns of the $T^{\prime}$ triplets $\phi$, $\zeta$ and $\xi$ in (2.8) are also consistent with the scalar potential minimization equations.

\section{References}

1. A. Abada, D. Das, A. Vicente, C. Weiland, JHEP 1209, 015 (2012). https://doi.org/10.1007/JHEP09(2012)015. arXiv:1206.6497 [hep-ph]

2. F. Deppisch, J.W.F. Valle, Phys. Rev. D 72, 036001 (2005). https:// doi.org/10.1103/PhysRevD.72.036001. arXiv:hep-ph/0406040

3. A. Abada, M.E. Krauss, W. Porod, F. Staub, A. Vicente, C. Weiland, JHEP 1411, 048 (2014). https://doi.org/10.1007/ JHEP11(2014)048. arXiv:1408.0138 [hep-ph]

4. A. Abada, M. Lucente, Nucl. Phys. B 885, 651 (2014). https://doi. org/10.1016/j.nuclphysb.2014.06.003. arXiv:1401.1507 [hep-ph]

5. A. Abada, T. Toma, JHEP 1608, 079 (2016). https://doi.org/10. 1007/JHEP08(2016)079. arXiv:1605.07643 [hep-ph]

6. A. Abada, Á. Hernández-Cabezudo, X. Marcano, JHEP 1901, 041 (2019). https://doi.org/10.1007/JHEP01(2019)041. arXiv:1807.01331 [hep-ph]

7. H. Georgi, A. Pais, Phys. Rev. D 19, 2746 (1979). https://doi.org/ 10.1103/PhysRevD.19.2746

8. J.W.F. Valle, M. Singer, Phys. Rev. D 28, 540 (1983). https://doi. org/10.1103/PhysRevD.28.540

9. F. Pisano, V. Pleitez, Phys. Rev. D 46, 410 (1992). https://doi.org/ 10.1103/PhysRevD.46.410. arXiv:hep-ph/9206242

10. R. Foot, O.F. Hernandez, F. Pisano, V. Pleitez, Phys. Rev. D 47, 4158 (1993). https://doi.org/10.1103/PhysRevD.47.4158. arXiv:hep-ph/9207264

11. P.H. Frampton, Phys. Rev. Lett. 69, 2889 (1992). https://doi.org/ 10.1103/PhysRevLett.69.2889

12. H.N. Long, Phys. Rev. D 54, 4691 (1996). https://doi.org/10.1103/ PhysRevD.54.4691. arXiv:hep-ph/9607439

13. H.N. Long, Phys. Rev. D 53, 437 (1996). https://doi.org/10.1103/ PhysRevD.53.437. arXiv:hep-ph/9504274

14. R. Foot, H.N. Long, T.A. Tran, Phys. Rev. D 50(1), R34 (1994). https://doi.org/10.1103/PhysRevD.50.R34. arXiv:hep-ph/9402243 
15. A.E.Carcamo Hernandez, R. Martinez, F. Ochoa, Phys. Rev. D 73, 035007 (2006). https://doi.org/10.1103/PhysRevD.73. 035007. arXiv:hep-ph/0510421

16. P.V. Dong, H.N. Long, D.V. Soa, V.V. Vien, Eur. Phys. J. C 71, 1544 (2011). https://doi.org/10.1140/epjc/s10052-011-1544-2. arXiv:1009.2328 [hep-ph]

17. P.V. Dong, L.T. Hue, H.N. Long, D.V. Soa, Phys. Rev. D 81, 053004 (2010). https://doi.org/10.1103/PhysRevD.81.053004. arXiv: 1001.4625 [hep-ph]

18. P.V. Dong, H.N. Long, C.H. Nam, V.V. Vien, Phys. Rev. D 85, 053001 (2012). https://doi.org/10.1103/PhysRevD.85.053001. arXiv:1111.6360 [hep-ph]

19. R.H. Benavides, W.A. Ponce, Y. Giraldo, Phys. Rev. D 82, 013004 (2010). https://doi.org/10.1103/PhysRevD.82.013004. arXiv: 1006.3248 [hep-ph]

20. P.V. Dong, H.N. Long, H.T. Hung, Phys. Rev. D 86, 033002 (2012). https://doi.org/10.1103/PhysRevD.86.033002. arXiv: 1205.5648 [hep-ph]

21. D.T. Huong, L.T. Hue, M.C. Rodriguez, H.N. Long, Nucl. Phys. B 870, 293 (2013). https://doi.org/10.1016/j.nuclphysb.2013.01. 016. arXiv:1210.6776 [hep-ph]

22. P.T. Giang, L.T. Hue, D.T. Huong, H.N. Long, Nucl. Phys. B 864, 85 (2012). https://doi.org/10.1016/j.nuclphysb.2012.06.008. arXiv: 1204.2902 [hep-ph]

23. D.T. Binh, L.T. Hue, D.T. Huong, H.N. Long, Eur. Phys. J. C 74(5), 2851 (2014). https://doi.org/10.1140/epjc/s10052-014-2851-1. arXiv:1308.3085 [hep-ph]

24. A.E.Carcamo Hernandez, R. Martinez, F. Ochoa, Phys. Rev. D 87(7), 075009 (2013). https://doi.org/10.1103/PhysRevD.87. 075009. arXiv:1302.1757 [hep-ph]

25. A.E.Cárcamo Hernández, R. Martinez, F. Ochoa, Eur. Phys. J. C 76(11), 634 (2016). https://doi.org/10.1140/epjc/ s10052-016-4480-3. arXiv:1309.6567 [hep-ph]

26. A.E.Cárcamo Hernández, R. Martinez, J. Nisperuza, Eur. Phys. J. C 75(2), 72 (2015). https://doi.org/10.1140/epjc/ s10052-015-3278-z. arXiv:1401.0937 [hep-ph]

27. A.E.Cárcamo Hernández, E.Cataño Mur, R. Martinez, Phys. Rev. D 90(7), 073001 (2014). https://doi.org/10.1103/PhysRevD.90. 073001. arXiv:1407.5217 [hep-ph]

28. C. Kelso, H.N. Long, R. Martinez, F.S. Queiroz, Phys. Rev. D 90(11), 113011 (2014). https://doi.org/10.1103/PhysRevD.90. 113011. arXiv:1408.6203 [hep-ph]

29. V.V. Vien, H.N. Long, JHEP 1404, 133 (2014). https://doi.org/10. 1007/JHEP04(2014)133. arXiv:1402.1256 [hep-ph]

30. V.Q. Phong, H.N. Long, V.T. Van, L.H. Minh, Eur. Phys. J. C 75(7), 342 (2015). https://doi.org/10.1140/epjc/ s10052-015-3550-2. arXiv:1409.0750 [hep-ph]

31. V.Q. Phong, H.N. Long, V.T. Van, N.C. Thanh, Phys. Rev. D 90(8), 085019 (2014). https://doi.org/10.1103/PhysRevD.90. 085019. [arXiv:1408.5657 [hep-ph]]

32. S.M. Boucenna, S. Morisi, J.W.F. Valle, Phys. Rev. D 90(1), 013005 (2014). https://doi.org/10.1103/PhysRevD.90.013005. arXiv: 1405.2332 [hep-ph]

33. G. De Conto, A.C.B. Machado, V. Pleitez, Phys. Rev. D 92(7), 075031 (2015). https://doi.org/10.1103/PhysRevD.92.075031. arXiv: 1505.01343 [hep-ph]

34. S.M. Boucenna, J.W.F. Valle, A. Vicente, Phys. Rev. D 92(5), 053001 (2015). https://doi.org/10.1103/PhysRevD.92.053001. arXiv:1502.07546 [hep-ph]

35. S.M. Boucenna, S. Morisi, A. Vicente, Phys. Rev. D 93(11), 115008 (2016). https://doi.org/10.1103/PhysRevD.93.115008. arXiv: 1512.06878 [hep-ph]

36. R.H. Benavides, L.N. Epele, H. Fanchiotti, C.G. Canal, W.A. Ponce, Adv. High Energy Phys. 2015, 813129 (2015). https:// doi.org/10.1155/2015/813129. arXiv:1503.01686 [hep-ph]
37. A.E.Cárcamo Hernández, R. Martinez, Nucl. Phys. B 905, 337 (2016). https://doi.org/10.1016/j.nuclphysb.2016.02.025. arXiv:1501.05937 [hep-ph]

38. L.T. Hue, H.N. Long, T.T. Thuc, T.Phong Nguyen, Nucl. Phys. B 907, 37 (2016). https://doi.org/10.1016/j.nuclphysb.2016.03.034. arXiv:1512.03266 [hep-ph]

39. A.E.C. Hernández, I. Nišandžić, Eur. Phys. J. C 76(7), 380 (2016). https://doi.org/10.1140/epjc/s10052-016-4230-6. arXiv:1512.07165 [hep-ph]

40. R.M. Fonseca, M. Hirsch, JHEP 1608, 003 (2016). https://doi. org/10.1007/JHEP08(2016)003. arXiv:1606.01109 [hep-ph]

41. R.M. Fonseca, M. Hirsch, Phys. Rev. D 94(11), 115003 (2016). $\quad$ https://doi.org/10.1103/PhysRevD.94.115003. arXiv:1607.06328 [hep-ph]

42. F.F. Deppisch, C. Hati, S. Patra, U. Sarkar, J.W.F. Valle, Phys. Lett. B 762, 432 (2016). https://doi.org/10.1016/j.physletb.2016. 10.002. arXiv: 1608.05334 [hep-ph]

43. M. Reig, J.W.F. Valle, C.A. Vaquera-Araujo, Phys. Rev. D 94(3), 033012 (2016). https://doi.org/10.1103/PhysRevD.94.033012. arXiv:1606.08499 [hep-ph]

44. A.E.Cárcamo Hernández, S. Kovalenko, H.N. Long, I. Schmidt, JHEP 1807, 144 (2018). https://doi.org/10.1007/ JHEP07(2018)144. arXiv:1705.09169 [hep-ph]

45. A.E.Cárcamo Hernández, H.N. Long, J. Phys. G 45(4), 045001 (2018). https://doi.org/10.1088/1361-6471/aaace7. arXiv:1705.05246 [hep-ph]

46. C. Hati, S. Patra, M. Reig, J.W.F. Valle, C.A. Vaquera-Araujo, Phys. Rev. D 96(1), 015004 (2017). https://doi.org/10.1103/ PhysRevD.96.015004. arXiv:1703.09647 [hep-ph]

47. E.R. Barreto, A.G. Dias, J. Leite, C.C. Nishi, R.L.N. Oliveira, W.C. Vieira, Phys. Rev. D 97(5), 055047 (2018). https://doi.org/ 10.1103/PhysRevD.97.055047. arXiv:1709.09946 [hep-ph]

48. A.E.Cárcamo Hernández, H.N. Long, V.V. Vien, Eur. Phys. J. C 78(10), 804 (2018). https://doi.org/10.1140/epjc/ s10052-018-6284-0. arXiv:1803.01636 [hep-ph]

49. V.V. Vien, H.N. Long, A.E.Cárcamo Hernández, Mod. Phys. Lett. A 34(01), 1950005 (2019). https://doi.org/10.1142/ S0217732319500056. arXiv:1812.07263 [hep-ph]

50. A.G. Dias, J. Leite, D.D. Lopes, C.C. Nishi, Phys. Rev. D 98(11), 115017 (2018). https://doi.org/10.1103/PhysRevD.98. 115017. arXiv: 1810.01893 [hep-ph]

51. M.M. Ferreira, T.B. de Melo, S. Kovalenko, P.R.D. Pinheiro, F.S. Queiroz. arXiv:1903.07634 [hep-ph]

52. D.T. Huong, D.N. Dinh, L.D. Thien, P. Van Dong, JHEP 1908, 051 (2019). https://doi.org/10.1007/JHEP08(2019)051. arXiv: 1906.05240 [hep-ph]

53. A. E. Cárcamo Hernández, N. A. Pérez-Julve, Y. H. Velásquez, arXiv:1907.13083 [hep-ph]

54. C.A. de Sousa Pires, O.P. Ravinez, Phys. Rev. D 58, 035008 (1998). https://doi.org/10.1103/PhysRevD.58.035008. arXiv:hep-ph/9803409 [Phys. Rev. D 58, 35008 (1998)]

55. P.V. Dong, H.N. Long, Int. J. Mod. Phys. A 21, 6677 (2006). $\quad$ https://doi.org/10.1142/S0217751X06035191. arXiv:hep-ph/0507155

56. J.C. Montero, V. Pleitez, O. Ravinez, Phys. Rev. D 60, 076003 (1999). https://doi.org/10.1103/PhysRevD.60.076003. arXiv:hep-ph/9811280

57. J.C. Montero, C.C. Nishi, V. Pleitez, O. Ravinez, M.C. Rodriguez, Phys. Rev. D 73, 016003 (2006). https://doi.org/10.1103/ PhysRevD.73.016003. arXiv:hep-ph/0511100

58. P.B. Pal, Phys. Rev. D 52, 1659 (1995). https://doi.org/10.1103/ PhysRevD.52.1659. arXiv:hep-ph/9411406

59. A.G. Dias, V. Pleitez, M.D. Tonasse, Phys. Rev. D 67, 095008 (2003). https://doi.org/10.1103/PhysRevD.67.095008. arXiv:hep-ph/0211107 
60. A.G. Dias, V. Pleitez, Phys. Rev. D 69, 077702 (2004). https:// doi.org/10.1103/PhysRevD.69.077702. arXiv:hep-ph/0308037

61. A.G. Dias, C.A. de S. Pires, P. S. Rodrigues da Silva, Phys. Rev. D 68, 115009 (2003). https://doi.org/10.1103/PhysRevD.68. 115009. arXiv:hep-ph/0309058

62. J .K. Mizukoshi, C .A. de S. Pires, F .S. Queiroz, P .S. Rodrigues da Silva, Phys. Rev. D 83, 065024 (2011). https://doi.org/10.1103/ PhysRevD.83.065024. arXiv:1010.4097 [hep-ph]

63. A.G. Dias, C.A. de S. Pires, P.S. Rodrigues da Silva, Phys. Rev. D 82, 035013 (2010) https://doi.org/10.1103/PhysRevD.82. 035013. arXiv:1003.3260 [hep-ph]

64. J.D. Ruiz-Alvarez, C.A. de S. Pires, F.S. Queiroz, D. Restrepo, P.S. Rodrigues da Silva, Phys. Rev. D 86, 075011 (2012) https://doi. org/10.1103/PhysRevD.86.075011. arXiv:1206.5779 [hep-ph]

65. D. Cogollo, A.X. Gonzalez-Morales, F.S. Queiroz, P.R. Teles, JCAP 1411(11), 002 (2014). https://doi.org/10.1088/1475-7516/ 2014/11/002. arXiv:1402.3271 [hep-ph]

66. A. Aranda, C.D. Carone, R.F. Lebed, Phys. Rev. D 62, 016009 (2000). https://doi.org/10.1103/PhysRevD.62.016009. arXiv:hep-ph/0002044

67. F. Feruglio, C. Hagedorn, Y. Lin, L. Merlo, Nucl. Phys. B 775, 120 (2007) Erratum: [Nucl. Phys. B 836, 127 (2010)] https://doi. org/10.1016/j.nuclphysb.2007.04.002, https://doi.org/10.1016/j. nuclphysb.2010.04.018. arXiv:hep-ph/0702194

68. S. Sen, Phys. Rev. D 76, 115020 (2007). https://doi.org/10.1103/ PhysRevD.76.115020. arXiv:0710.2734 [hep-ph]

69. A. Aranda, Phys. Rev. D 76, 111301 (2007). https://doi.org/10. 1103/PhysRevD.76.111301. arXiv:0707.3661 [hep-ph]

70. M.C. Chen, K.T. Mahanthappa, Phys. Lett. B 652, 34 (2007). https://doi.org/10.1016/j.physletb.2007.06.064. arXiv:0705.0714 [hep-ph]

71. D.A. Eby, P.H. Frampton, S. Matsuzaki, Phys. Lett. B 671, 386 (2009). https://doi.org/10.1016/j.physletb.2008.11.074. arXiv:0810.4899 [hep-ph]

72. P.H. Frampton, T.W. Kephart, S. Matsuzaki, Phys. Rev. D 78, 073004 (2008). https://doi.org/10.1103/PhysRevD.78.073004. arXiv:0807.4713 [hep-ph]

73. P.H. Frampton, S. Matsuzaki, Mod. Phys. Lett. A 24, 429 (2009). https://doi.org/10.1142/S0217732309030229. arXiv:0807.4785 [hep-ph]

74. D.A. Eby, P.H. Frampton, S. Matsuzaki, Phys. Rev. D 80, 053007 (2009). https://doi.org/10.1103/PhysRevD.80.053007. arXiv:0907.3425 [hep-ph]

75. P.H. Frampton, S. Matsuzaki, Phys. Lett. B 679, 347 (2009). https://doi.org/10.1016/j.physletb.2009.08.001. arXiv:0902.1140 [hep-ph]

76. L. Merlo, S. Rigolin, B. Zaldivar, JHEP 1111, 047 (2011). https:// doi.org/10.1007/JHEP11(2011). arXiv:1108.1795 [hep-ph]

77. D.A. Eby, P.H. Frampton, X.G. He, T.W. Kephart, Phys. Rev. D 84, 037302 (2011). https://doi.org/10.1103/PhysRevD.84. 037302. arXiv:1103.5737 [hep-ph]

78. D.A. Eby, P.H. Frampton, Phys. Lett. B 713, 249 (2012). https:// doi.org/10.1016/j.physletb.2012.06.004. arXiv:1111.4938 [hep$\mathrm{ph}]$

79. M.C. Chen, K.T. Mahanthappa. arXiv:1107.3856 [hep-ph]

80. A. Meroni, S.T. Petcov, M. Spinrath, Phys. Rev. D 86, 113003 (2012). https://doi.org/10.1103/PhysRevD.86.113003. arXiv:1205.5241 [hep-ph]

81. P.H. Frampton, C.M. Ho, T.W. Kephart, Phys. Rev. D 89(2), 027701 (2014). https://doi.org/10.1103/PhysRevD.89.027701. arXiv: 1305.4402 [hep-ph]

82. M.C. Chen, J. Huang, K.T. Mahanthappa, A.M. Wijangco, JHEP 1310, 112 (2013). https://doi.org/10.1007/JHEP10(2013)112. arXiv:1307.7711 [hep-ph]
83. I. Girardi, A. Meroni, S.T. Petcov, M. Spinrath, JHEP 1402, 050 (2014). https://doi.org/10.1007/JHEP02(2014)050. arXiv:1312.1966 [hep-ph]

84. C.D. Carone, S. Chaurasia, S. Vasquez, Phys. Rev. D 95(1), 015025 (2017). https://doi.org/10.1103/PhysRevD.95.015025. arXiv:1611.00784 [hep-ph]

85. C.D. Carone, M. Merchand, Phys. Rev. D 100(3), 035006 (2019). $\quad$ https://doi.org/10.1103/PhysRevD.100.035006. arXiv:1904.11059 [hep-ph]

86. E. Ma, G. Rajasekaran, Phys. Rev. D 64, 113012 (2001). https:// doi.org/10.1103/PhysRevD.64.113012. arXiv:hep-ph/0106291

87. X.G. He, Y.Y. Keum, R.R. Volkas, JHEP 0604, 039 (2006). https://doi.org/10.1088/1126-6708/2006/04/039. arXiv:hep-ph/0601001

88. F. Feruglio, C. Hagedorn, Y. Lin, L. Merlo, Nucl. Phys. B 809, 218 (2009). https://doi.org/10.1016/j.nuclphysb.2008.10. 002. arXiv:0807.3160 [hep-ph]

89. F. Feruglio, C. Hagedorn, Y. Lin, L. Merlo, Nucl. Phys. B 832, 251 (2010). https://doi.org/10.1016/j.nuclphysb.2010.02. 010. arXiv:0911.3874 [hep-ph]

90. M.C. Chen, S.F. King, JHEP 0906, 072 (2009). https://doi.org/ 10.1088/1126-6708/2009/06/072. arXiv:0903.0125 [hep-ph]

91. I. de Medeiros Varzielas, L. Merlo, JHEP 1102, 062 (2011). https://doi.org/10.1007/JHEP02(2011)062. arXiv:1011.6662 [hep-ph]

92. G. Altarelli, F. Feruglio, L. Merlo, E. Stamou, JHEP 1208, 021 (2012). https://doi.org/10.1007/JHEP08(2012)021. arXiv: 1205.4670 [hep-ph]

93. Y.H. Ahn, S.K. Kang, Phys. Rev. D 86, 093003 (2012). https://doi. org/10.1103/PhysRevD.86.093003. arXiv:1203.4185 [hep-ph]

94. N. Memenga, W. Rodejohann, H. Zhang, Phys. Rev. D 87(5), 053021 (2013). https://doi.org/10.1103/PhysRevD.87.053021. arXiv:1301.2963 [hep-ph]

95. R.Gonzalez Felipe, H. Serodio, J.P. Silva, Phys. Rev. D 88(1), 015015 (2013). https://doi.org/10.1103/PhysRevD.88.015015. arXiv: 1304.3468 [hep-ph]

96. I. de Medeiros Varzielas, D. Pidt, JHEP 1303, 065 (2013). https:// doi.org/10.1007/JHEP03(2013)065. arXiv:1211.5370 [hep-ph]

97. H. Ishimori, E. Ma, Phys. Rev. D 86, 045030 (2012). https://doi. org/10.1103/PhysRevD.86.045030. arXiv:1205.0075 [hep-ph]

98. S.F. King, S. Morisi, E. Peinado, J.W.F. Valle, Phys. Lett. B 724, 68 (2013). https://doi.org/10.1016/j.physletb.2013.05.067. arXiv: 1301.7065 [hep-ph]

99. A.E.Carcamo Hernandez, I. de Medeiros Varzielas, S.G. Kovalenko, H. Päs, I. Schmidt, Phys. Rev. D 88(7), 076014 (2013). https://doi.org/10.1103/PhysRevD.88.076014. arXiv:1307.6499 [hep-ph]

100. K.S. Babu, E. Ma, J.W.F. Valle, Phys. Lett. B $\mathbf{5 5 2}$ 207 (2003). https://doi.org/10.1016/S0370-2693(02)03153-2. [arXiv:hep-ph/0206292]

101. G. Altarelli, F. Feruglio, Nucl. Phys. B 741, 215 (2006). https://doi.org/10.1016/j.nuclphysb.2006.02.015. arXiv:hep-ph/0512103

102. S. Gupta, A.S. Joshipura, K.M. Patel, Phys. Rev. D 85 , 031903 (2012). https://doi.org/10.1103/PhysRevD.85.031903. arXiv:1112.6113 [hep-ph]

103. S. Morisi, M. Nebot, K.M. Patel, E. Peinado, J.W.F. Valle, Phys. Rev. D 88, 036001 (2013). https://doi.org/10.1103/PhysRevD.88. 036001. arXiv:1303.4394 [hep-ph]

104. G. Altarelli, F. Feruglio, Nucl. Phys. B 720, 64 (2005). https://doi. org/10.1016/j.nuclphysb.2005.05.005. arXiv:hep-ph/0504165

105. A. Kadosh, E. Pallante, JHEP 1008, 115 (2010). https://doi.org/ 10.1007/JHEP08(2010)115. arXiv:1004.0321 [hep-ph]

106. A. Kadosh, JHEP 1306, 114 (2013). https://doi.org/10.1007/ JHEP06(2013)114. arXiv:1303.2645 [hep-ph] 
107. F. del Aguila, A. Carmona, J. Santiago, JHEP 1008, 127 (2010). https://doi.org/10.1007/JHEP08(2010)127. arXiv:1001.5151 [hep-ph]

108. M.D. Campos, A.E.Cárcamo Hernández, S. Kovalenko, I. Schmidt, E. Schumacher, Phys. Rev. D 90(1), 016006 (2014). https://doi.org/10.1103/PhysRevD.90.016006. arXiv:1403.2525 [hep-ph]

109. V.V. Vien, H.N. Long, Int. J. Mod. Phys. A 30(21), 1550117 (2015). https://doi.org/10.1142/S0217751X15501171. arXiv: 1405.4665 [hep-ph]

110. A.S. Joshipura, K.M. Patel, Phys. Lett. B 749, 159 (2015). https:// doi.org/10.1016/j.physletb.2015.07.062. arXiv:1507.01235 [hep$\mathrm{ph}]$

111. B. Karmakar, A. Sil, Phys. Rev. D 96(1), 015007 (2017). https:// doi.org/10.1103/PhysRevD.96.015007. arXiv:1610.01909 [hep$\mathrm{ph}]$

112. P. Chattopadhyay, K.M. Patel, Nucl. Phys. B 921, 487 (2017). https://doi.org/10.1016/j.nuclphysb.2017.06.008. arXiv:1703.09541 [hep-ph]

113. E. Ma, G. Rajasekaran, EPL 119(3), 31001 (2017). https://doi. org/10.1209/0295-5075/119/31001. arXiv:1708.02208 [hep-ph]

114. S.Centelles Chuliá, R. Srivastava, J.W.F. Valle, Phys. Lett. B 773, 26 (2017). https://doi.org/10.1016/j.physletb.2017.07.065. arXiv: 1706.00210 [hep-ph]

115. F. Björkeroth, E.J. Chun, S.F. King, Phys. Lett. B 777, 428 (2018). https://doi.org/10.1016/j.physletb.2017.12.058. arXiv:1711.05741 [hep-ph]

116. R. Srivastava, C.A. Ternes, M. Tórtola, J.W.F. Valle, Phys. Lett. B 778, 459 (2018). https://doi.org/10.1016/j.physletb.2018.01.014. arXiv:1711.10318 [hep-ph]

117. D. Borah, B. Karmakar, Phys. Lett. B 780, 461 (2018). https://doi. org/10.1016/j.physletb.2018.03.047. arXiv:1712.06407 [hep-ph]

118. A.S. Belyaev, S.F. King, P.B. Schaefers, Phys. Rev. D 97(11), 115002 (2018). https://doi.org/10.1103/PhysRevD.97.115002. arXiv:1801.00514 [hep-ph]

119. A.E.Cárcamo Hernández, S.F. King, Phys. Rev. D 99(9), 095003 (2019). https://doi.org/10.1103/PhysRevD.99.095003. arXiv:1803.07367 [hep-ph]

120. R. Srivastava, C.A. Ternes, M. Tórtola, J.W.F. Valle, Phys. Rev. D 97(9), 095025 (2018). https://doi.org/10.1103/PhysRevD.97. 095025. arXiv:1803.10247 [hep-ph]

121. L.M.G. De La Vega, R. Ferro-Hernandez, E. Peinado, Phys. Rev. D 99(5), 055044 (2019). https://doi.org/10.1103/PhysRevD.99. 055044. arXiv:1811.10619 [hep-ph]

122. D. Borah, B. Karmakar, Phys. Lett. B 789, 59 (2019). https://doi. org/10.1016/j.physletb.2018.12.006. arXiv:1806.10685 [hep-ph]

123. S. Pramanick. arXiv:1903.04208 [hep-ph]

124. A.E. Cárcamo Hernández, J. Marchant González, U.J. SaldañaSalazar, arXiv:1904.09993 [hep-ph]

125. A.E. Cárcamo Hernández, M. González, N.A. Neill. arXiv:1906.00978 [hep-ph]

126. G.J. Ding, S.F. King, X.G. Liu. arXiv:1907.11714 [hep-ph]

127. G. Altarelli, F. Feruglio, L. Merlo, JHEP 0905, 020 (2009). https:// doi.org/10.1088/1126-6708/2009/05/020. arXiv:0903.1940 [hep-ph]

128. F. Bazzocchi, L. Merlo, S. Morisi, Phys. Rev. D 80, 053003 (2009). https://doi.org/10.1103/PhysRevD.80.053003. arXiv:0902.2849 [hep-ph]

129. F. Bazzocchi, L. Merlo, S. Morisi, Nucl. Phys. B 816, 204 (2009). https://doi.org/10.1016/j.nuclphysb.2009.03.005. arXiv:0901.2086 [hep-ph]

130. R. de Adelhart Toorop, F. Bazzocchi, L. Merlo, JHEP 1008, 001 (2010). https://doi.org/10.1007/JHEP08(2010)001. arXiv: 1003.4502 [hep-ph]

131. K.M. Patel, Phys. Lett. B 695, 225 (2011). https://doi.org/10. 1016/j.physletb.2010.11.024. arXiv:1008.5061 [hep-ph]
132. S. Morisi, K.M. Patel, E. Peinado, Phys. Rev. D 84, 053002 (2011). https://doi.org/10.1103/PhysRevD.84.053002. arXiv:1107.0696 [hep-ph]

133. R.N. Mohapatra, C.C. Nishi, Phys. Rev. D 86, 073007 (2012). https://doi.org/10.1103/PhysRevD.86.073007. arXiv:1208.2875 [hep-ph]

134. P.S.Bhupal Dev, B. Dutta, R.N. Mohapatra, M. Severson, Phys. Rev. D 86, 035002 (2012). https://doi.org/10.1103/PhysRevD.86. 035002. [arXiv:1202.4012 [hep-ph]]

135. I. de Medeiros Varzielas, L. Lavoura, J. Phys. G 40, 085002 (2013). https://doi.org/10.1088/0954-3899/40/8/085002. arXiv:1212.3247 [hep-ph]

136. G.J. Ding, S.F. King, C. Luhn, A.J. Stuart, JHEP 1305, 084 (2013). https://doi.org/10.1007/JHEP05(2013)084. arXiv:1303.6180 [hep-ph]

137. H. Ishimori, Y. Shimizu, M. Tanimoto, A. Watanabe, Phys. Rev. D 83, 033004 (2011). https://doi.org/10.1103/PhysRevD.83. 033004. arXiv:1010.3805 [hep-ph]

138. G.J. Ding, Y.L. Zhou, Nucl. Phys. B 876, 418 (2013). https://doi. org/10.1016/j.nuclphysb.2013.08.011. arXiv:1304.2645 [hep-ph]

139. C. Hagedorn, M. Serone, JHEP 1110, 083 (2011). https://doi.org/ 10.1007/JHEP10(2011). arXiv:1106.4021 [hep-ph]

140. M.D. Campos, A.E.Cárcamo Hernández, H. Päs, E. Schumacher, Phys. Rev. D 91(11), 116011 (2015). https://doi.org/10.1103/ PhysRevD.91.116011. arXiv:1408.1652 [hep-ph]

141. V.V. Vien, H.N. Long, D.P. Khoi, Int. J. Mod. Phys. A 30(17), 1550102 (2015). https://doi.org/10.1142/S0217751X1550102X. arXiv:1506.06063 [hep-ph]

142. F.J. de Anda, S.F. King, E. Perdomo, JHEP 1712, 075 (2017) Erratum: [JHEP 1904, 069 (2019)] https://doi.org/10.1007/ JHEP12(2017)075, https://doi.org/10.1007/JHEP04(2019)069. arXiv: 1710.03229 [hep-ph]

143. F.J. de Anda, S.F. King, JHEP 1807, 057 (2018). https://doi.org/ 10.1007/JHEP07(2018)057. arXiv:1803.04978 [hep-ph]

144. A.E. Cárcamo Hernández, S.F. King. arXiv:1903.02565 [hep-ph]

145. P.T. Chen, G.J. Ding, S.F. King, C.C. Li. arXiv:1906.11414 [hep$\mathrm{ph}]$

146. I. De Medeiros Varzielas, S.F. King, Y.L. Zhou, arXiv:1906.02208 [hep-ph]

147. I. De Medeiros Varzielas, M. Levy, Y.L. Zhou. arXiv:1903.10506 [hep-ph]

148. S.F. King, Y.L. Zhou. arXiv:1908.02770 [hep-ph]

149. G.C. Branco, J.M. Gerard, W. Grimus, Phys. Lett. 136B, 383 (1984). https://doi.org/10.1016/0370-2693(84)92024-0

150. I. de Medeiros Varzielas, S.F. King, G.G. Ross, Phys. Lett. B 648, 201 (2007). https://doi.org/10.1016/j.physletb.2007.03.009. arXiv:hep-ph/0607045

151. E. Ma, Phys. Lett. B 660, 505 (2008). https://doi.org/10.1016/j. physletb.2007.12.060. arXiv:0709.0507 [hep-ph]

152. I. de Medeiros Varzielas, D. Emmanuel-Costa, P. Leser, Phys. Lett. B 716, 193 (2012). https://doi.org/10.1016/j.physletb.2012. 08.008. arXiv:1204.3633 [hep-ph]

153. G. Bhattacharyya, I. de Medeiros Varzielas, P. Leser, Phys. Rev. Lett. 109, 241603 (2012). https://doi.org/10.1103/PhysRevLett. 109.241603. arXiv:1210.0545 [hep-ph]

154. P.M. Ferreira, W. Grimus, L. Lavoura, P.O. Ludl, JHEP 1209, 128 (2012). https://doi.org/10.1007/JHEP09(2012)128. arXiv:1206.7072 [hep-ph]

155. E. Ma, Phys. Lett. B 723, 161 (2013). https://doi.org/10.1016/j. physletb.2013.05.011. arXiv:1304.1603 [hep-ph]

156. C.C. Nishi, Phys. Rev. D 88(3), 033010 (2013). https://doi.org/ 10.1103/PhysRevD.88.033010. arXiv:1306.0877 [hep-ph]

157. I. de Medeiros Varzielas, D. Pidt, J. Phys. G 41, 025004 (2014). https://doi.org/10.1088/0954-3899/41/2/025004. arXiv:1307.0711 [hep-ph] 
158. A. Aranda, C. Bonilla, S. Morisi, E. Peinado, J.W.F. Valle, Phys. Rev. D 89(3), 033001 (2014). https://doi.org/10.1103/PhysRevD. 89.033001. arXiv:1307.3553 [hep-ph]

159. P.F. Harrison, R. Krishnan, W.G. Scott, Int. J. Mod. Phys. A 29(18), 1450095 (2014). https://doi.org/10.1142/ S0217751X1450095X. arXiv:1406.2025 [hep-ph]

160. E. Ma, A. Natale, Phys. Lett. B 734, 403 (2014). https://doi.org/ 10.1016/j.physletb.2014.05.070. arXiv:1403.6772 [hep-ph]

161. M. Abbas, S. Khalil, Phys. Rev. D 91(5), 053003 (2015). https://doi.org/10.1103/PhysRevD.91.053003. arXiv:1406.6716 [hep-ph]

162. M. Abbas, S. Khalil, A. Rashed, A. Sil, Phys. Rev. D 93(1), 013018 (2016). https://doi.org/10.1103/PhysRevD.93.013018. arXiv:1508.03727 [hep-ph]

163. I. de Medeiros Varzielas, JHEP 1508, 157 (2015). https://doi.org/ 10.1007/JHEP08(2015)157. arXiv:1507.00338 [hep-ph]

164. F. Björkeroth, F.J. de Anda, I. de Medeiros Varzielas, S.F. King, Phys. Rev. D 94(1), 016006 (2016). https://doi.org/10.1103/ PhysRevD.94.016006. arXiv:1512.00850 [hep-ph]

165. P. Chen, G.J. Ding, A.D. Rojas, C.A. Vaquera-Araujo, J.W.F. Valle, JHEP 1601, 007 (2016). https://doi.org/10.1007/ JHEP01(2016)007. arXiv:1509.06683 [hep-ph]

166. V.V. Vien, A.E.Cárcamo Hernández, H.N. Long, Nucl. Phys. B 913, 792 (2016). https://doi.org/10.1016/j.nuclphysb.2016.10. 010. arXiv:1601.03300 [hep-ph]

167. A.E.Cárcamo Hernández, H.N. Long, V.V. Vien, Eur. Phys. J. C 76(5), 242 (2016). https://doi.org/10.1140/epjc/ s10052-016-4074-0. arXiv:1601.05062 [hep-ph]

168. A.E.Cárcamo Hernández, S. Kovalenko, J.W.F. Valle, C.A. Vaquera-Araujo, JHEP 1707, 118 (2017). https://doi.org/10. 1007/JHEP07(2017)118. arXiv:1705.06320 [hep-ph]

169. I. de Medeiros Varzielas, G.G. Ross, J. Talbert, JHEP 1803, 007 (2018). https://doi.org/10.1007/JHEP03(2018)007. arXiv: 1710.01741 [hep-ph]

170. N. Bernal, A.E.Cárcamo Hernández, I. de Medeiros Varzielas, S. Kovalenko, JHEP 1805, 053 (2018). https://doi.org/10.1007/ JHEP05(2018)053. arXiv:1712.02792 [hep-ph]

171. I. De Medeiros Varzielas, M.L. López-Ibáñez, A. Melis, O. Vives, JHEP 1809, 047 (2018). https://doi.org/10.1007/ JHEP09(2018)047. arXiv:1807.00860 [hep-ph]

172. A.E.Cárcamo Hernández, S. Kovalenko, J.W.F. Valle, C.A. Vaquera-Araujo, JHEP 1902, 065 (2019). https://doi.org/10. 1007/JHEP02(2019)065. arXiv:1811.03018 [hep-ph]

173. A.E.Cárcamo Hernández, J.C. Gómez-Izquierdo, S. Kovalenko, M. Mondragón, Nucl. Phys. B 946, 114688 (2019). https://doi. org/10.1016/j.nuclphysb.2019.114688. arXiv:1810.01764 [hep$\mathrm{ph}]$

174. F. Björkeroth, I. de Medeiros Varzielas, M.L. López-Ibáñez, A. Melis, Ó. Vives. arXiv:1904.10545 [hep-ph]

175. A. Davidson, K.C. Wali, Phys. Rev. Lett. 59, 393 (1987). https:// doi.org/10.1103/PhysRevLett.59.393

176. Z.G. Berezhiani, R. Rattazzi, Phys. Lett. B 279, 124 (1992). https://doi.org/10.1016/0370-2693(92)91851-Y

177. I.S. Sogami, T. Shinohara, Prog. Theor. Phys. 86, 1031 (1991). https://doi.org/10.1143/PTP.86.1031

178. R.N. Mohapatra, J.W.F. Valle, Phys. Rev. D 34, 1642 (1986). https://doi.org/10.1103/PhysRevD.34.1642

179. M.C. Gonzalez-Garcia, J.W.F. Valle, Phys. Lett. B 216, 360 (1989). https://doi.org/10.1016/0370-2693(89)91131-3

180. E.K. Akhmedov, M. Lindner, E. Schnapka, J.W.F. Valle, Phys. Rev. D 53, 2752 (1996). https://doi.org/10.1103/PhysRevD.53. 2752. arXiv:hep-ph/9509255

181. E.K. Akhmedov, M. Lindner, E. Schnapka, J.W.F. Valle, Phys. Lett. B 368, 270 (1996). https://doi.org/10.1016/ 0370-2693(95)01504-3. arXiv:hep-ph/9507275
182. M. Malinsky, J.C. Romao, J.W.F. Valle, Phys. Rev. Lett. 95, 161801 (2005). https://doi.org/10.1103/PhysRevLett.95.161801. arXiv:hep-ph/0506296

183. M. Malinsky, T. Ohlsson, Zz Xing, H. Zhang, Phys. Lett. B 679, 242 (2009). https://doi.org/10.1016/j.physletb.2009.07.038. arXiv:0905.2889 [hep-ph]

184. C.D. Froggatt, H.B. Nielsen, Nucl. Phys. B 147, 277 (1979). https://doi.org/10.1016/0550-3213(79)90316-X

185. V.T.N. Huyen, H.N. Long, T.T. Lam, V.Q. Phong, Commun. Phys. 24(2), 97 (2014). https://doi.org/10.15625/0868-3166/24/ 2/3774. arXiv:1210.5833 [hep-ph]

186. C. Salazar, R.H. Benavides, W.A. Ponce, E. Rojas, JHEP 1507, 096 (2015). https://doi.org/10.1007/JHEP07(2015)096. arXiv:1503.03519 [hep-ph]

187. R. Martinez, F. Ochoa, Phys. Rev. D 77, 065012 (2008). https:// doi.org/10.1103/PhysRevD.77.065012. arXiv:0802.0309 [hep$\mathrm{ph}]$

188. A.J. Buras, F. De Fazio, J. Girrbach, JHEP 1402, 112 (2014). https://doi.org/10.1007/JHEP02(2014)112. arXiv:1311.6729 [hep-ph]

189. A.J. Buras, F. De Fazio, J. Girrbach-Noe, JHEP 1408, 039 (2014). https://doi.org/10.1007/JHEP08(2014)039. arXiv:1405.3850 [hep-ph]

190. A.J. Buras, F. De Fazio, J. Girrbach, M.V. Carlucci, JHEP 1302, 023 (2013). https://doi.org/10.1007/JHEP02(2013)023. arXiv:1211.1237 [hep-ph]

191. R.A. Diaz, R. Martinez, F. Ochoa, Phys. Rev. D 69, 095009 (2004). https://doi.org/10.1103/PhysRevD.69.095009. arXiv:hep-ph/0309280

192. H.N. Long, N.V. Hop, L.T. Hue, N.H. Thao, A.E.Cárcamo Hernández, Phys. Rev. D 100(1), 015004 (2019). https://doi.org/ 10.1103/PhysRevD.100.015004. arXiv:1810.00605 [hep-ph]

193. M.A. Perez, G. Tavares-Velasco, J.J. Toscano, Phys. Rev. D 69, 115004 (2004). https://doi.org/10.1103/PhysRevD.69.115004. arXiv:hep-ph/0402156

194. M. Aaboud et al., ATLAS Collaboration. JHEP 1801, 055 (2018). https://doi.org/10.1007/JHEP01(2018)055. arXiv:1709.07242 [hep-ex]

195. K. Bora, Horizon 2, 112 (2013). arXiv:1206.5909 [hep-ph]

196. Zz Xing, H. Zhang, S. Zhou, Phys. Rev. D 77, 113016 (2008). https://doi.org/10.1103/PhysRevD.77.113016. arXiv:0712.1419 [hep-ph]

197. C. Patrignani et al., [Particle Data Group], Chin. Phys. C 40(10), 100001 (2016). https://doi.org/10.1088/1674-1137/40/ $10 / 100001$

198. M.E. Catano, R. Martinez, F. Ochoa, Phys. Rev. D 86, 073015 (2012). https://doi.org/10.1103/PhysRevD.86.073015. arXiv:1206.1966 [hep-ph]

199. P.S.Bhupal Dev, R. Franceschini, R.N. Mohapatra, Phys. Rev. D 86, 093010 (2012). https://doi.org/10.1103/PhysRevD.86. 093010. arXiv:1207.2756 [hep-ph]

200. A. Das, N. Okada, Phys. Rev. D 88, 113001 (2013). https://doi. org/10.1103/PhysRevD.88.113001. arXiv:1207.3734 [hep-ph]

201. A. Das, P.S.Bhupal Dev, N. Okada, Phys. Lett. B 735, 364 (2014). https://doi.org/10.1016/j.physletb.2014.06.058. arXiv: 1405.0177 [hep-ph]

202. A. Das, P. Konar, S. Majhi, JHEP 1606, 019 (2016). https://doi. org/10.1007/JHEP06(2016)019. arXiv:1604.00608 [hep-ph]

203. A. Das, P. Konar, A. Thalapillil, JHEP 1802, 083 (2018). https:// doi.org/10.1007/JHEP02(2018)083. arXiv:1709.09712 [hep-ph]

204. A. Das, N. Okada, Phys. Lett. B 774, 32 (2017). https://doi.org/ 10.1016/j.physletb.2017.09.042. arXiv:1702.04668 [hep-ph]

205. A. Das, P.S.B. Dev, C.S. Kim, Phys. Rev. D 95(11), 115013 (2017). https://doi.org/10.1103/PhysRevD.95.115013. arXiv:1704.00880 [hep-ph] 
206. A. Das, Y. Gao, T. Kamon, Eur. Phys. J. C 79(5), 424 (2019). https://doi.org/10.1140/epjc/s10052-019-6937-7. arXiv: 1704.00881 [hep-ph]

207. A. Das, S. Jana, S. Mandal, S. Nandi, Phys. Rev. D 99(5), 055030 (2019). https://doi.org/10.1103/PhysRevD.99.055030. arXiv:1811.04291 [hep-ph]

208. A. Das, Adv. High Energy Phys. 2018, 9785318 (2018). https:// doi.org/10.1155/2018/9785318. arXiv:1803.10940 [hep-ph]

209. A. Bhardwaj, A. Das, P. Konar, A. Thalapillil, arXiv:1801.00797 [hep-ph]

210. J.C. Helo, H. Li, N.A. Neill, M. Ramsey-Musolf, J.C. Vasquez, Phys. Rev. D 99(5), 055042 (2019). https://doi.org/10.1103/ PhysRevD.99.055042. arXiv:1812.01630 [hep-ph]

211. S. Pascoli, R. Ruiz, C. Weiland, JHEP 1906, 049 (2019). https:// doi.org/10.1007/JHEP06(2019)049. arXiv:1812.08750 [hep-ph]

212. P.F. de Salas, D.V. Forero, C.A. Ternes, M. Tortola, J.W.F. Valle, Phys. Lett. B 782, 633 (2018). https://doi.org/10.1016/j.physletb. 2018.06.019. arXiv:1708.01186 [hep-ph]

213. M.A. Shifman, A.I. Vainshtein, M.B. Voloshin, V.I. Zakharov, Sov. J. Nucl. Phys. 30, 711 (1979). [Yad. Fiz. 30, 1368 (1979)]

214. M.B. Gavela, G. Girardi, C. Malleville, P. Sorba, Nucl. Phys. B 193, 257 (1981). https://doi.org/10.1016/0550-3213(81)90529-0

215. P. Kalyniak, R. Bates, J.N. Ng, Phys. Rev. D 33, 755 (1986). https://doi.org/10.1103/PhysRevD.33.755

216. A. Djouadi, Phys. Rept. 459, 1 (2008). https://doi.org/10.1016/j. physrep.2007.10.005. arXiv:hep-ph/0503173

217. W.J. Marciano, C. Zhang, S. Willenbrock, Phys. Rev. D 85, 013002 (2012). https://doi.org/10.1103/PhysRevD.85.013002. arXiv: 1109.5304 [hep-ph]

218. L. Wang, X.F. Han, Phys. Rev. D 86, 095007 (2012). https://doi. org/10.1103/PhysRevD.86.095007. arXiv:1206.1673 [hep-ph]

219. J.F. Gunion, H.E. Haber, G.L. Kane, S. Dawson, Front. Phys. 80, $1(2000)$

220. M. Spira, Fortsch. Phys. 46, 203 (1998). https://doi.org/10.1002/ (SICI)1521-3978(199804)46:3<203::AID-PROP203>3.0.CO; 2-4 [arXiv:hep-ph/9705337]

221. A.E.Carcamo Hernandez, C.O. Dib, A.R. Zerwekh, Eur. Phys. J. C 74, 2822 (2014). https://doi.org/10.1140/epjc/ s10052-014-2822-6. arXiv:1304.0286 [hep-ph]
222. G. Bhattacharyya, D. Das, Phys. Rev. D 91, 015005 (2015). https://doi.org/10.1103/PhysRevD.91.015005. arXiv:1408.6133 [hep-ph]

223. E.C.F.S. Fortes, A.C.B. Machado, J. Montaño, V. Pleitez, J. Phys. G 42(11), 115001 (2015). https://doi.org/10.1088/0954-3899/42/ 11/115001. arXiv:1408.0780 [hep-ph]

224. A.E. Carcamo Hernandez, C.O. Dib, A.R. Zerwekh, Nucl. Part. Phys. Proc. 267-269, 35 (2015) https://doi.org/10.1016/j. nuclphysbps.2015.10.079. arXiv:1503.08472 [hep-ph]

225. A.E. Cárcamo Hernández, C.O. Dib, A. R. Zerwekh. arXiv: 1506.03631 [hep-ph]

226. V. Khachatryan et al., [CMS Collaboration], Eur. Phys. J. C 74(10), 3076 (2014) https://doi.org/10.1140/epjc/ s10052-014-3076-z. arXiv:1407.0558 [hep-ex]

227. G. Aad et al., [ATLAS Collaboration], Phys. Rev. D 90(11), 112015 (2014) https://doi.org/10.1103/PhysRevD.90. 112015 [arXiv: 1408.7084 [hep-ex]]

228. A. Ilakovac, A. Pilaftsis, Nucl. Phys. B 437, 491 (1995). https://doi.org/10.1016/0550-3213(94)00567-X. arXiv:hep-ph/9403398

229. M. Lindner, M. Platscher, F.S. Queiroz, Phys. Rept. 731, 1 (2018). https://doi.org/10.1016/j.physrep.2017.12.001. arXiv: 1610.06587 [hep-ph]

230. F.F. Deppisch, N. Desai, J.W.F. Valle, Phys. Rev. D 89(5), 051302 (2014). https://doi.org/10.1103/PhysRevD.89.051302. arXiv:1308.6789 [hep-ph]

231. J.A. Aguilar-Saavedra, F. Deppisch, O. Kittel, J.W.F. Valle, Phys. Rev. D 85, 091301 (2012). https://doi.org/10.1103/PhysRevD.85. 091301. arXiv:1203.5998 [hep-ph]

232. S.P. Das, F.F. Deppisch, O. Kittel, J.W.F. Valle, Phys. Rev. D 86, 055006 (2012). https://doi.org/10.1103/PhysRevD.86. 055006. arXiv:1206.0256 [hep-ph]

233. H. Ishimori, T. Kobayashi, H. Ohki, Y. Shimizu, H. Okada, M. Tanimoto, Prog. Theor. Phys. Suppl. 183, 1 (2010). https://doi. org/10.1143/PTPS.183.1. arXiv:1003.3552 [hep-th] 